\title{
AN IMPROVED INTERPOLATION SCHEME FOR FINITE VOLUME SIMULATIONS ON UNSTRUCTURED MESHES
}

\author{
SAMUEL K. M. CHENOWETH, JULIO SORIA, AND ANDREW OOI
}

\begin{abstract}
The unstructured bilinear interpolation scheme of Kim and Choi (2000) is claimed to be second order accurate on the basis of empirical results from finite volume simulations of the incompressible Navier-Stokes equations. In this paper, the scheme is analysed theoretically, and is shown to be only first order accurate for function interpolation and zeroth order accurate for spatial derivative approximation, in the general case. A number of special cases exist, however, where higher order accuracy may be obtained, and these are identified in this paper. Since the mesh used by Kim and Choi to demonstrate the accuracy of their scheme was one of these special cases, this explains their results. Finally, an improved version of Kim and Choi's scheme is presented, which is shown to be truly second order accurate for function interpolation and first order accurate for spatial derivative approximation.
\end{abstract}

\section{INTRODUCTION}

Finite volume simulations are a popular method of solving partial differential equations, especially those relating to fluid flow phenomena, owing to their flux conservative properties. Increasingly, such finite volume solvers are being developed for use with unstructured meshes, since this allows problems with complex domain geometry to be simulated, as well as allowing a high degree of flexibility in mesh design. While unstructured meshes have these advantages, their use necessitates the employment of an interpolation scheme, so that the fluxes may be computed. In an element centred finite volume scheme, for example, the simulation variables are tracked at each element centroid of the mesh, and some interpolation scheme must be used to approximate the values and spatial derivatives of these variables at each of the edge midpoints, so that the fluxes between each element and its neighbours may be computed. On an unstructured mesh, the interpolation is complicated by the fact that there may be an arbitrary geometric and/or topological relationship between each edge midpoint and the surrounding element centroids which form the stencil for the interpolation.

A variety of schemes exist which are capable of handling interpolations on irregular data, for instance, Sheppard interpolation [9], radial basis functions [4] or moving least squares [7]. The above techniques were originally developed for handling scattered data, where there is no mesh topology at all. While they may easily be adapted to interpolations on unstructured meshes (e.g., by [1]), they are not

Received by the editor June 24, 2010 and, in revised form, May 29, 2011.

2010 Mathematics Subject Classification. Primary 65D05.

Key words and phrases. Interpolation, unstructured meshes.

The authors would like to acknowledge the support of the Australian Research Council for this research through grant DP0556098. 
necessarily the best tools for the task as they do not make full use of the mesh topology.

One interpolation scheme which does make effective use of the mesh topology is an unstructured version of bilinear interpolation, which evolved through the work of Davidson [3, Lai [6] and principally Kim and Choi [5. In its original form, the bilinear interpolation scheme provides a very simple way of interpolating the value of a function and its first order spatial derivatives on a regular, rectangular mesh. In essence, interpolation in multiple dimensions is achieved through a sequence of linear interpolations along straight line segments, until an interpolated value is obtained for the desired location. Although this scheme is very well known and in common use, it is only more recently that it has been adapted to unstructured meshes. Davidson's approach was to perform a weighted linear interpolation between the two element centroids adjacent to an edge, and then use this value as an approximation to the value at the edge midpoint. The deficiency of this method is that the straight line segment connecting the two adjacent element centroids does not necessarily intersect the edge at its midpoint, and so the interpolated value is really for the wrong point in space. It was shown by Kim and Choi that the function interpolation is only first order accurate if this issue is not taken into account.

Having identified the problem with Davidson's scheme, Kim and Choi attempted to fix it, developing a scheme which is claimed to be second order accurate. While that scheme is not named in their paper, it is referred to in this paper as "unstructured bilinear interpolation" for convenience. Their innovation was the addition of an extra term to the equation for the interpolation, which allows for the fact that on an unstructured mesh, the midpoint of an edge may not be coincident with a line connecting the adjacent element centroids. However, their claim to second order accuracy is based on empirical data, not on an analytical proof; that is, they used their unstructured bilinear scheme in a two-dimensional incompressible NavierStokes solver and simulated the problem of a decaying vortex in a square cavity, which has a known analytical solution. By studying the error trend as the mesh was progressively refined, they were able to demonstrate second order accuracy. Using empirical techniques is acceptable, in the absence of a rigourous analytical proof, however, their method demonstrates second order spatial accuracy for the overall solver, as opposed to the interpolation scheme in isolation. Even if the order of accuracy is not affected by other aspects of the solver, this still fails to distinguish between the order of accuracy of the function and spatial derivative interpolations, which in general are different. Moreover, their mesh refinement study was limited to one particular mesh design. It is thus not clear whether second order accuracy also applies to a wider range of unstructured mesh topologies.

In this paper it is shown that, in general, Kim and Choi's scheme is in fact only first order accurate for function interpolation and zeroth order accurate for spatial derivative approximation. Kim and Choi's results may be explained by the existence of several special cases of mesh geometry where second order function interpolation is available, one of which corresponds to the mesh that they used for their testing. There are also special cases where first or second order accurate spatial derivative approximation is possible. The principal special cases are identified in this paper, and these represent the full set known to the authors.

Finally, this paper presents an improved version of Kim and Choi's interpolation scheme, which is named "hybrid unstructured bilinear interpolation". This 
improved scheme is truly second order accurate for function interpolation and first order accurate for spatial derivative approximation, in the general case. (Special cases are also identified where the spatial derivatives are second order accurate.) The order of accuracy of the hybrid unstructured bilinear interpolation scheme is proven, and the improvement over Kim and Choi's scheme is demonstrated empirically. Note that for the sake of simplicity and brevity, the scope of the discussion in this paper is mostly limited to the interpolation schemes as they apply in the interior of the domain. The methods used by Kim and Choi [5] for incorporating boundary conditions are not repeated in this paper, and the incorporation of boundary conditions in the hybrid unstructured bilinear scheme is only explained briefly.

1.1. Another perspective: Diamond-cell schemes. Both the unstructured bilinear scheme used by Kim and Choi and the hybrid unstructured bilinear scheme presented in this paper are variants of the diamond-cell scheme for interpolation. In the diamond-cell scheme, the function and spatial derivative interpolations at the midpoint of a mesh edge are obtained on the basis of the function values at the two adjacent element centroids, as well as the function values at the two end nodes of the edge. These four points form a diamond with the edge midpoint at its centre, which is the origin of the scheme's name. Conceptually, the diamond-cell scheme determines the gradient vector of the function on the basis of two differences, namely the difference between the two function values at the adjacent element centroids and the difference between the two interpolated function values at the end nodes of the interpolation edge. Since the displacement vectors for these two differences are approximately perpendicular in most cases, this method is usually well posed. Having obtained the gradient, the interpolated value of the function at the edge midpoint can then be "extrapolated" from the value of the function at one of the adjacent element centroids.

A diamond-cell scheme was also used by Coudiere et al. 2], although Kim and Choi did not appear to be aware of this earlier work and did not use this terminology. While the various diamond-cell schemes are fundamentally similar, they differ principally in the means by which the interpolated function values are obtained for the end nodes. While Kim and Choi's unstructured bilinear scheme used a simple weighted mean for their nodal interpolations, the hybrid unstructured bilinear scheme of this paper and the scheme used by Coudiere et al. both use linear interpolation based on a best fit, which is more accurate. However, it should be noted that the hybrid unstructured bilinear scheme differs from the scheme of Coudiere et al., in that Coudiere et al. compute their convective flux using function values from the upwind adjacent element centroid, and so do not need to consider the interpolation of the function at edge midpoints. Both the hybrid and conventional versions of the unstructured bilinear scheme, by contrast, interpolate the function on the edge midpoints using approximately balanced stencils, in order to minimise dissipative effects when used in finite volume solvers. In addition, the three schemes vary in the way that interpolations are obtained on the mesh boundaries, although a detailed discussion of this issue is beyond the scope of this paper.

1.2. Outline of this paper. Section 2 presents a derivation and analysis of the general diamond-cell scheme. The error terms that are obtained are crucial for identifying the special cases that can exist, and the authors believe this is the first 
time that this has been done comprehensively. (The previous analysis by Coudiere et al. is broadly consistent with the results of this paper for the general case, but did not consider the difference between the order of accuracy of the two components of the gradient that can occur in certain special cases. Hence, they detected the special case of parallelogram meshes, but omitted some other special cases.) Since the order of accuracy of the diamond-cell scheme is dependent on the accuracy of the interpolated function values at the end nodes, this needs to be considered next. Section 3.2 explains the nodal interpolation scheme used by Kim and Choi [5] in their unstructured bilinear interpolation, analysing the scheme's accuracy in the general case and in one special case. Section 3.3 explains the improved nodal interpolation scheme used in the hybrid unstructured bilinear scheme suggested in this paper. The accuracy of this nodal interpolation scheme is also provided for the general case. (There are no special cases known to the authors.) In Section 4 , the order of accuracy of the difference between two nodal interpolations is determined for the general case, and two special cases are identified. The results of all these sections are then combined in Section 5, explaining how the actual order of accuracy that applies in any given case may be determined. Finally, Section 6 provides some numerical results that confirm some of the theoretical findings of this paper.

\section{DiAMOND-CELL SCHEMES FOR INTERPOLATION}

2.1. Geometry and notation. Figure 1 shows two neighbouring unstructured mesh elements, with the (internal) interpolation edge separating them. Although triangles are drawn, either or both of the elements could be quadrilaterals without changing any of the following analysis. Concave quadrilaterals are disallowed, however. Points $e_{1}$ and $e_{2}$ are the centroids of the elements and points $n_{a}$ and $n_{b}$ are the (internal) nodes at either end of the interpolation edge. Point $\mathrm{P}_{\mathrm{i}}$ is at the intersection of the interpolation edge with the line connecting the two element centroids. $\Theta$ is the angle between this line and the normal to the interpolation edge. Point $\mathrm{P}_{\mathrm{m}}$ lies at the midpoint of the interpolation edge, which is where the value of $f$ and its spatial derivatives are to be interpolated. Also marked are the coordinate axes for the $(\delta, \varepsilon)$ edge coordinate system, whose origin is at the midpoint of the interpolation edge and whose axes are normal and parallel to the edge. The dimensions are shown on the diagram, with the convention that $\delta_{1}$ and $\delta_{2}$ represent dimensions that are normal to the interpolation edge while $\varepsilon_{1}, \varepsilon_{2}, \varepsilon_{\mathrm{m}}$ and $\varepsilon_{\mathrm{ab}}$ represent dimensions that are parallel to the interpolation edge. Note that these are dimensions, not coordinates in $(\delta, \varepsilon)$ space, so that $\delta_{1}>0$ (and $\delta_{2}>0$ ) even though the $\delta$ coordinate of point $\mathrm{e}_{1}$ is negative. Nevertheless, $\varepsilon_{1}, \varepsilon_{2}$ and $\varepsilon_{\mathrm{m}}$ are signed quantities, so that they are positive in the configuration shown but would be negative if their sense were opposite. Further, the signs of $\varepsilon_{1}$ and $\varepsilon_{2}$ are linked, so that in any configuration they must be both positive, both negative or both zero. It should also be noted that the dimensions are not measured from the origin of the $(\delta, \varepsilon)$ edge coordinate system, but are exactly as indicated on Figure 1, $\varepsilon_{\mathrm{ab}}$, for example, is the length of the interpolation edge, and so neither end of this dimension is at the origin. A relation between some of these dimensions may be obtained using similar triangles, which is

$$
\delta_{1} \varepsilon_{2}-\delta_{2} \varepsilon_{1}=0 .
$$

This relation is required for some of the algebraic manipulations in this section. 


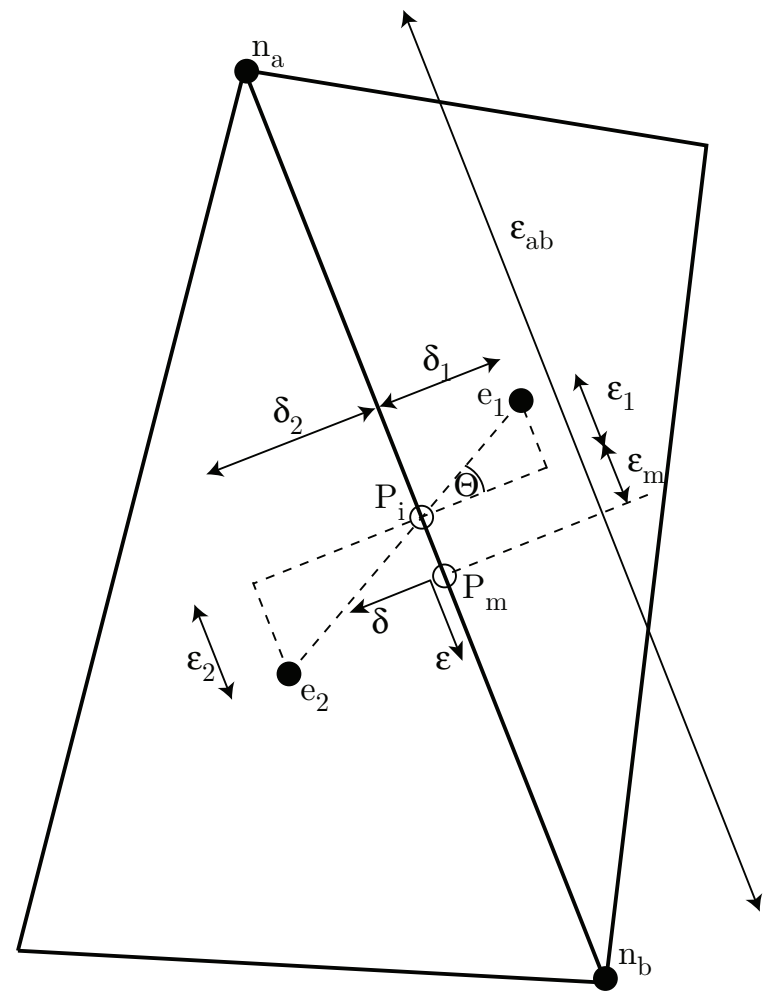

FiguRE 1. Schematic of the unstructured bilinear scheme.

Table 1 explains the different values of $f$ and its derivatives referred to in the rest of this paper.

TABLE 1. Notation for function and derivative values

\begin{tabular}{|l|ll|l|l|}
\hline Location & \multicolumn{2}{|c|}{$\begin{array}{l}\text { Function Value } \\
\text { Exact Interpolated }\end{array}$} & $\begin{array}{l}\text { Derivative Perpendicular to Edge } \\
\text { Exact Interpolated }\end{array}$ & $\begin{array}{l}\text { Derivative Parallel to Edge } \\
\text { Exact Interpolated }\end{array}$ \\
\hline Edge Midpoint $\mathrm{P}_{\mathrm{m}}$ & $f_{\mathrm{m}} \quad f_{\mathrm{m}}^{*}$ & $\frac{\partial f_{\mathrm{m}}}{\partial \delta} \quad \frac{\partial f_{\mathrm{m}}^{*}}{\partial \delta}$ & $\frac{\partial f_{\mathrm{m}}}{\partial \varepsilon} \quad \frac{\partial f_{\mathrm{m}}^{*}}{\partial \varepsilon}$ \\
\hline End Node $\mathrm{n}_{\mathrm{a}}$ & $f_{\mathrm{a}} \quad f_{\mathrm{a}}^{*}$ & $\frac{\partial f_{\mathrm{a}}}{\partial \delta}$ & $\frac{\partial f_{\mathrm{a}}}{\partial \varepsilon}$ \\
\hline End Node $\mathrm{n}_{\mathrm{b}}$ & $f_{\mathrm{b}} \quad f_{\mathrm{b}}^{*}$ & $\frac{\partial f_{\mathrm{b}}}{\partial \delta}$ & $\frac{\partial f_{\mathrm{b}}}{\partial \varepsilon}$ \\
\hline Element Centroid $\mathrm{e}_{1}$ & $f_{\mathrm{e} 1}$ & & \\
\hline Element Centroid $\mathrm{e}_{2}$ & $f_{\mathrm{e} 2}$ & & \\
\hline
\end{tabular}

2.2. Derivation of the scheme. The value of $f$ at the nodes may be expressed using Taylor's expansions centred on the midpoint of the edge, so that

$$
f_{\mathrm{b}}=f_{\mathrm{m}}+\left(\frac{\varepsilon_{\mathrm{ab}}}{2}\right) \frac{\partial f_{\mathrm{m}}}{\partial \varepsilon}+\frac{1}{2 !} \cdot\left(\frac{\varepsilon_{\mathrm{ab}}}{2}\right)^{2} \frac{\partial^{2} f_{\mathrm{m}}}{\partial \varepsilon^{2}}+\frac{1}{3 !} \cdot\left(\frac{\varepsilon_{\mathrm{ab}}}{2}\right)^{3} \frac{\partial^{3} f_{\mathrm{m}}}{\partial \varepsilon^{3}}+O\left(\Delta^{4}\right)
$$

and

$$
f_{\mathrm{a}}=f_{\mathrm{m}}-\left(\frac{\varepsilon_{\mathrm{ab}}}{2}\right) \frac{\partial f_{\mathrm{m}}}{\partial \varepsilon}+\frac{1}{2 !} \cdot\left(\frac{\varepsilon_{\mathrm{ab}}}{2}\right)^{2} \frac{\partial^{2} f_{\mathrm{m}}}{\partial \varepsilon^{2}}-\frac{1}{3 !} \cdot\left(\frac{\varepsilon_{\mathrm{ab}}}{2}\right)^{3} \frac{\partial^{3} f_{\mathrm{m}}}{\partial \varepsilon^{3}}+O\left(\Delta^{4}\right)
$$


and therefore

$$
f_{\mathrm{b}}-f_{\mathrm{a}}=\varepsilon_{\mathrm{ab}} \frac{\partial f_{\mathrm{m}}}{\partial \varepsilon}+O\left(\Delta^{3}\right) .
$$

Now in diamond-cell interpolation schemes exact values are not available at the nodes, so these must be interpolated from the values at the surrounding element centroids. If an interpolation scheme of order of accuracy $p$ is used, then

$$
f_{\mathrm{b}}^{*}=f_{\mathrm{b}}+O\left(\Delta^{p}\right)
$$

and

$$
f_{\mathrm{a}}^{*}=f_{\mathrm{a}}+O\left(\Delta^{p}\right)
$$

where the asterisk indicates an interpolated rather than exact value. Hence the difference between the interpolated nodal values is

$$
f_{\mathrm{b}}^{*}-f_{\mathrm{a}}^{*}=f_{\mathrm{b}}-f_{\mathrm{a}}+O\left(\Delta^{P}\right),
$$

where $P \geq p$. Note that this takes into account the possibility that some of the error terms could be canceled in the subtraction. Such effects are investigated in Section 4. Substituting (2.4) into (2.7) yields

$$
f_{\mathrm{b}}^{*}-f_{\mathrm{a}}^{*}=\varepsilon_{\mathrm{ab}} \frac{\partial f_{\mathrm{m}}}{\partial \varepsilon}+O\left(\Delta^{P}\right)+O\left(\Delta^{3}\right) .
$$

The values at the element centroids may also be expressed using Taylor's expansions centred on the midpoint of the edge, so that

$$
\begin{aligned}
f_{\mathrm{e} 2}= & f_{\mathrm{m}}+\left(\varepsilon_{2}-\varepsilon_{\mathrm{m}}\right) \frac{\partial f_{\mathrm{m}}}{\partial \varepsilon}+\delta_{2} \frac{\partial f_{\mathrm{m}}}{\partial \delta}+\frac{1}{2 !} \cdot\left(\varepsilon_{2}-\varepsilon_{\mathrm{m}}\right)^{2} \frac{\partial^{2} f_{\mathrm{m}}}{\partial \varepsilon^{2}} \\
& +\frac{2}{2 !} \cdot\left(\varepsilon_{2}-\varepsilon_{\mathrm{m}}\right) \delta_{2} \frac{\partial^{2} f_{\mathrm{m}}}{\partial \varepsilon \partial \delta}+\frac{1}{2 !} \cdot \delta_{2}^{2} \frac{\partial^{2} f_{\mathrm{m}}}{\partial \delta^{2}}+O\left(\Delta^{3}\right)
\end{aligned}
$$

and

$$
\begin{aligned}
f_{\mathrm{e} 1}= & f_{\mathrm{m}}-\left(\varepsilon_{1}+\varepsilon_{\mathrm{m}}\right) \frac{\partial f_{\mathrm{m}}}{\partial \varepsilon}-\delta_{1} \frac{\partial f_{\mathrm{m}}}{\partial \delta}+\frac{1}{2 !} \cdot\left(\varepsilon_{1}+\varepsilon_{\mathrm{m}}\right)^{2} \frac{\partial^{2} f_{\mathrm{m}}}{\partial \varepsilon^{2}} \\
& +\frac{2}{2 !} \cdot\left(\varepsilon_{1}+\varepsilon_{\mathrm{m}}\right) \delta_{1} \frac{\partial^{2} f_{\mathrm{m}}}{\partial \varepsilon \partial \delta}+\frac{1}{2 !} \cdot \delta_{1}^{2} \frac{\partial^{2} f_{\mathrm{m}}}{\partial \delta^{2}}+O\left(\Delta^{3}\right) .
\end{aligned}
$$

The difference between the values at the element centroids is then

$$
\begin{aligned}
f_{\mathrm{e} 2}-f_{\mathrm{e} 1}= & \left(\varepsilon_{2}+\varepsilon_{1}\right) \frac{\partial f_{\mathrm{m}}}{\partial \varepsilon}+\left(\delta_{2}+\delta_{1}\right) \frac{\partial f_{\mathrm{m}}}{\partial \delta} \\
& +\frac{1}{2 !}\left(\varepsilon_{2}+\varepsilon_{1}\right)\left(\varepsilon_{2}-\varepsilon_{1}-2 \varepsilon_{\mathrm{m}}\right) \frac{\partial^{2} f_{\mathrm{m}}}{\partial \varepsilon^{2}} \\
& +\frac{2}{2 !}\left[\delta_{2}\left(\varepsilon_{2}-\varepsilon_{\mathrm{m}}\right)-\delta_{1}\left(\varepsilon_{1}+\varepsilon_{\mathrm{m}}\right)\right] \frac{\partial^{2} f_{\mathrm{m}}}{\partial \varepsilon \partial \delta} \\
& +\frac{1}{2 !}\left(\delta_{2}+\delta_{1}\right)\left(\delta_{2}-\delta_{1}\right) \frac{\partial^{2} f_{\mathrm{m}}}{\partial \delta^{2}}+O\left(\Delta^{3}\right) .
\end{aligned}
$$

Note that the $O\left(\Delta^{3}\right)$ terms do not cancel in the subtraction, although they are omitted from this paper for clarity.

It is now useful to consider the conditions under which all the second order terms of (2.11) disappear. Using the fact that $\delta_{1}>0$ and $\delta_{2}>0$, it is clear that the last of the second order terms vanishes if and only if $\delta_{2}=\delta_{1}$. If this is the case, then (2.1) implies that $\varepsilon_{2}=\varepsilon_{1}$ also, and so the second of the second order terms vanishes if and only if $\varepsilon_{\mathrm{m}}=0$. With $\varepsilon_{2}=\varepsilon_{1}$ and $\varepsilon_{\mathrm{m}}=0$, the first of the second order 
terms vanishes too. Equation (2.11) can then be rewritten in a more useable form, in which the conditions under which the second order terms vanish can be clearly seen as follows:

$$
\begin{aligned}
f_{\mathrm{e} 2}-f_{\mathrm{e} 1}= & \left(\varepsilon_{2}+\varepsilon_{1}\right) \frac{\partial f_{\mathrm{m}}}{\partial \varepsilon}+\left(\delta_{2}+\delta_{1}\right) \frac{\partial f_{\mathrm{m}}}{\partial \delta} \\
& +O(\Delta)\left(\delta_{2}-\delta_{1}\right)+O(\Delta) \varepsilon_{\mathrm{m}}+O\left(\Delta^{3}\right) .
\end{aligned}
$$

Solving the system of equations consisting of (2.8) and (2.12) for the spatial derivatives yields

$$
\frac{\partial f_{\mathrm{m}}}{\partial \varepsilon}=\frac{f_{\mathrm{b}}^{*}-f_{\mathrm{a}}^{*}}{\varepsilon_{\mathrm{ab}}}+O\left(\Delta^{P-1}\right)+O\left(\Delta^{2}\right)
$$

and

$$
\begin{aligned}
\frac{\partial f_{\mathrm{m}}}{\partial \delta}= & \frac{f_{\mathrm{e} 2}-f_{\mathrm{e} 1}}{\delta_{2}+\delta_{1}}-\frac{\varepsilon_{2}+\varepsilon_{1}}{\delta_{2}+\delta_{1}} \cdot \frac{f_{\mathrm{b}}^{*}-f_{\mathrm{a}}^{*}}{\varepsilon_{\mathrm{ab}}} \\
& +\left(\varepsilon_{2}+\varepsilon_{1}\right) O\left(\Delta^{P-2}\right)+O(1)\left(\delta_{2}-\delta_{1}\right)+O(1) \varepsilon_{\mathrm{m}}+O\left(\Delta^{2}\right)
\end{aligned}
$$

The interpolated spatial derivatives $\frac{\partial f_{m}^{*}}{\partial \varepsilon}$ and $\frac{\partial f_{m}^{*}}{\partial \delta}$ can then be obtained using the above expressions, simply by ignoring the error terms (the terms involving $O(\ldots)$ ). Hence,

$$
\frac{\partial f_{\mathrm{m}}^{*}}{\partial \varepsilon}-\frac{\partial f_{\mathrm{m}}}{\partial \varepsilon}=O\left(\Delta^{P-1}\right)+O\left(\Delta^{2}\right)
$$

and

$$
\frac{\partial f_{\mathrm{m}}^{*}}{\partial \delta}-\frac{\partial f_{\mathrm{m}}}{\partial \delta}=\left(\varepsilon_{2}+\varepsilon_{1}\right) O\left(\Delta^{P-2}\right)+O(1)\left(\delta_{2}-\delta_{1}\right)+O(1) \varepsilon_{\mathrm{m}}+O\left(\Delta^{2}\right)
$$

Having derived the scheme for computing the spatial derivatives, it is now necessary to consider the interpolation of the function itself at the edge midpoint. To this end, equation (2.9) may be written more simply as

$$
f_{\mathrm{e} 2}=f_{\mathrm{m}}+\left(\varepsilon_{2}-\varepsilon_{\mathrm{m}}\right) \frac{\partial f_{\mathrm{m}}}{\partial \varepsilon}+\delta_{2} \frac{\partial f_{\mathrm{m}}}{\partial \delta}+O\left(\Delta^{2}\right)
$$

Note that this may be done without eliminating any special cases, as $\delta_{2}>0$ and so the last of the second order terms in (2.9) cannot vanish. Likewise, the equivalent expression for the other element centroid is

$$
f_{\mathrm{e} 1}=f_{\mathrm{m}}-\left(\varepsilon_{1}+\varepsilon_{\mathrm{m}}\right) \frac{\partial f_{\mathrm{m}}}{\partial \varepsilon}-\delta_{1} \frac{\partial f_{\mathrm{m}}}{\partial \delta}+O\left(\Delta^{2}\right)
$$


Computing $\delta_{1}$ multiplied by (2.17) plus $\delta_{2}$ multiplied by (2.18) yields

$$
\delta_{1} f_{\mathrm{e} 2}+\delta_{2} f_{\mathrm{e} 1}=\left(\delta_{1}+\delta_{2}\right) f_{\mathrm{m}}-\varepsilon_{\mathrm{m}}\left(\delta_{1}+\delta_{2}\right) \frac{\partial f_{\mathrm{m}}}{\partial \varepsilon}+O\left(\Delta^{3}\right) .
$$

By substituting (2.13) into (2.19), it may be shown that

$$
f_{\mathrm{m}}=\frac{\delta_{1} f_{\mathrm{e} 2}+\delta_{2} f_{\mathrm{e} 1}}{\delta_{1}+\delta_{2}}+\varepsilon_{\mathrm{m}} \cdot \frac{f_{\mathrm{b}}^{*}-f_{\mathrm{a}}^{*}}{\varepsilon_{\mathrm{ab}}}+\varepsilon_{\mathrm{m}} O\left(\Delta^{P-1}\right)+O\left(\Delta^{2}\right) .
$$

The interpolated value of the function can then be obtained using the above expression, simply by ignoring the error terms. Hence,

$$
f_{\mathrm{m}}^{*}-f_{\mathrm{m}}=\varepsilon_{\mathrm{m}} O\left(\Delta^{P-1}\right)+O\left(\Delta^{2}\right) .
$$

The accuracy of a diamond-cell scheme may be determined using (2.15), (2.16) and (2.21), where the orders of accuracy obtained depend on the order of accuracy of the nodal difference $(P)$ and on the geometrical properties of the interpolation edge. In particular, the conditions that can affect the order of accuracy are:

- When $\varepsilon_{\mathrm{m}}=0$. This means the straight line connecting the adjacent element centroids passes through the midpoint of the interpolation edge.

- When $\varepsilon_{2}+\varepsilon_{1}=0$. Since $\varepsilon_{1}$ and $\varepsilon_{2}$ have the same sign, this happens if and only if $\varepsilon_{2}=\varepsilon_{1}=0$. This means the straight line connecting the adjacent element centroids is normal to the interpolation edge.

- When $\delta_{1}=\delta_{2}$. This means the two adjacent element centroids are equidistant from the interpolation edge.

Figures 2, 3, and 4 are flowcharts that explain how the above theory may be used to determine the orders of accuracy, for $P=3, P=2$ and $P=1$, respectively. At each branch of the tree some new information becomes known, with the order of accuracy of the function and both spatial derivatives fully determined for each leaf. (Known information at any branch of the tree is passed on to all its sub-branches, and for the sake of brevity is not repeated.)

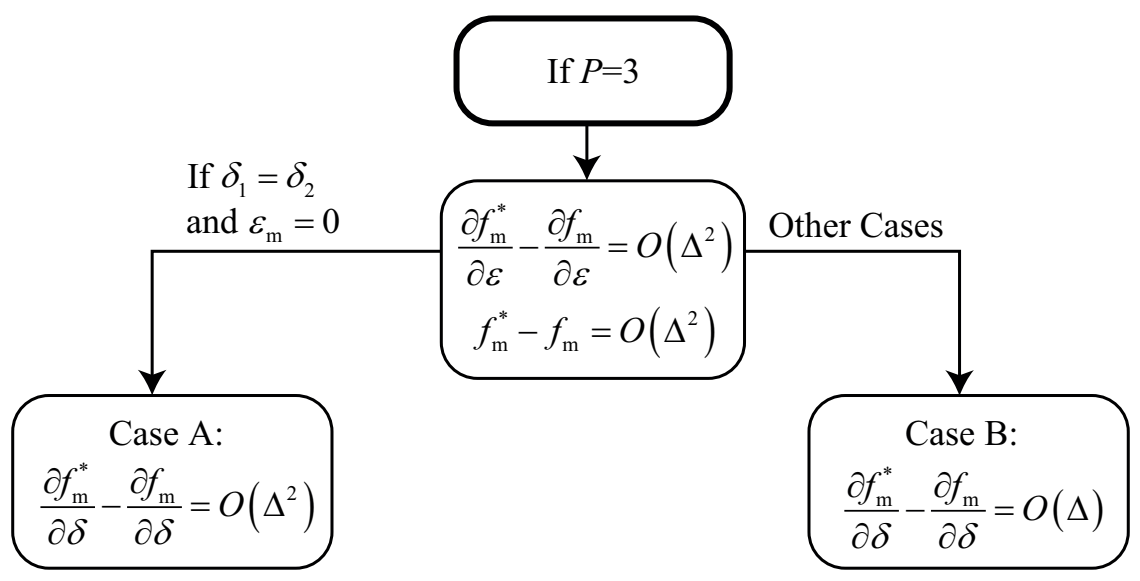

Figure 2. Flowchart for determining accuracy when $P=3$ 


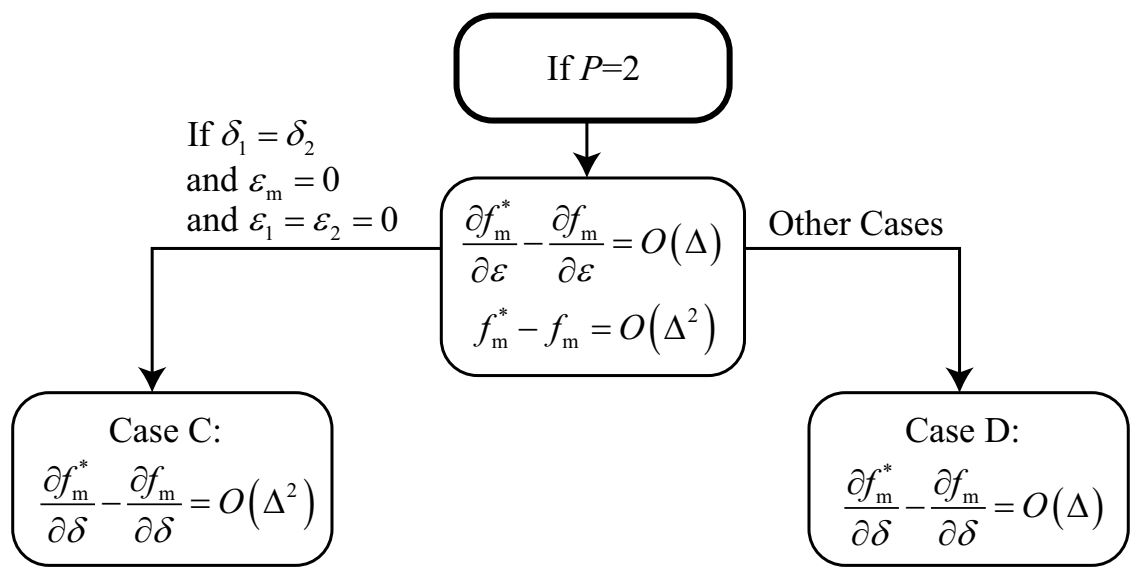

FiguRE 3. Flowchart for determining accuracy when $P=2$

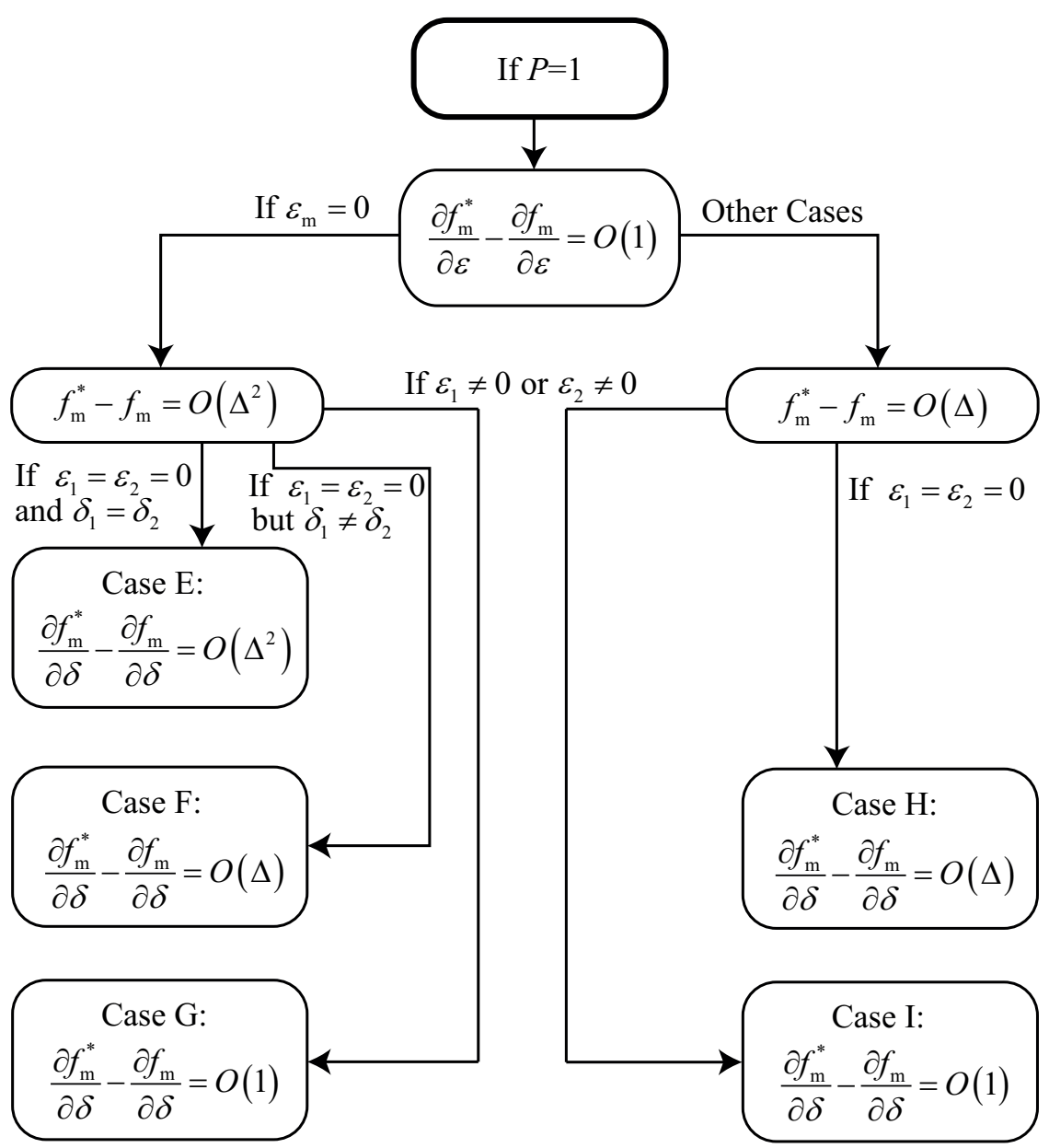

Figure 4. Flowchart for determining accuracy when $P=1$ 


\section{NODAL INTERPOLATION}

3.1. Accuracy analysis. This paper considers nodal interpolation schemes where the value of $f$ at each node is approximated as a weighted sum of the values of $f$ at the centroids of the elements surrounding it. Hence the interpolated value of $f$ at end node $\mathrm{n}_{\mathrm{b}}$ can be expressed as

$$
f_{\mathrm{b}}^{*}=\sum_{j}\left[q_{j \mathrm{~b}} f_{j \mathrm{~b}}\right]
$$

where $f_{j \mathrm{~b}}$ is the value of $f$ at the centroid of the $j$ th element surrounding node $\mathrm{n}_{\mathrm{b}}$ and $q_{j \mathrm{~b}}$ is its weight. It is possible to express $f_{j \mathrm{~b}}$ as a Taylor's series expansion about node $\mathrm{n}_{\mathrm{b}}$, so that

$$
f_{j \mathrm{~b}}=f_{\mathrm{b}}+\left(\varepsilon_{j \mathrm{~b}}-\frac{1}{2} \varepsilon_{\mathrm{ab}}\right) \frac{\partial f_{\mathrm{b}}}{\partial \varepsilon}+\delta_{j \mathrm{~b}} \frac{\partial f_{\mathrm{b}}}{\partial \delta}+O\left(\Delta^{2}\right)
$$

where $\delta_{j \mathrm{~b}}$ and $\varepsilon_{j \mathrm{~b}}$ are the coordinates of the $j$ th element centroid surrounding node $\mathrm{n}_{\mathrm{b}}$. Using (3.1) and (3.2), it may be shown that

$$
\begin{aligned}
f_{\mathrm{b}}^{*}-f_{\mathrm{b}}= & f_{\mathrm{b}}\left(\sum_{j}\left[q_{j \mathrm{~b}}\right]-1\right)+\frac{\partial f_{\mathrm{b}}}{\partial \varepsilon} \sum_{j}\left[q_{j \mathrm{~b}}\left(\varepsilon_{j \mathrm{~b}}-\frac{1}{2} \varepsilon_{\mathrm{ab}}\right)\right] \\
& +\frac{\partial f_{\mathrm{b}}}{\partial \delta} \sum_{j}\left[q_{j \mathrm{~b}} \delta_{j \mathrm{~b}}\right]+O\left(\Delta^{2}\right) .
\end{aligned}
$$

Hence the interpolation at node $\mathrm{n}_{\mathrm{b}}$ is second order accurate if $\sum_{j}\left[q_{j \mathrm{~b}}\right]=1$, $\sum_{j}\left[q_{j \mathrm{~b}}\left(\varepsilon_{j \mathrm{~b}}-\frac{1}{2} \varepsilon_{\mathrm{ab}}\right)\right]=0$ and $\sum_{j}\left[q_{j \mathrm{~b}} \delta_{j \mathrm{~b}}\right]=0$, but first order accurate if $\sum_{j}\left[q_{j \mathrm{~b}}\right]=1$ only.

In a similar manner, it may be shown that the interpolation at node $\mathrm{n}_{\mathrm{a}}$ is second order accurate if $\sum_{j}\left[q_{j \mathrm{a}}\right]=1, \sum_{j}\left[q_{j \mathrm{a}}\left(\varepsilon_{j \mathrm{a}}+\frac{1}{2} \varepsilon_{\mathrm{ab}}\right)\right]=0$ and $\sum_{j}\left[q_{j \mathrm{a}} \delta_{j \mathrm{a}}\right]=0$, but first order accurate if $\sum_{j}\left[q_{j a}\right]=1$ only.

\subsection{Nodal interpolation in Kim and Choi's unstructured bilinear scheme.}

In the interior of the mesh, the unstructured bilinear scheme of Kim and Choi [5] is simply the diamond-cell scheme presented earlier, with the following scheme used for the nodal interpolations. Kim and Choi's nodal interpolation is essentially a weighted mean of the $f$ values at the surrounding element centroids, with each weight proportional to the reciprocal of that element's area. Hence,

$$
q_{j \mathrm{~b}}=\frac{\left(\frac{1}{A_{j \mathrm{~b}}}\right)}{\sum_{k}\left[\frac{1}{A_{k \mathrm{~b}}}\right]},
$$

where $A_{j \mathrm{~b}}$ is the area of the $j$ th element surrounding node $\mathrm{n}_{\mathrm{b}}$ and the $k$ summation is over the set of elements surrounding the node. Obviously, a similar expression may be written for the interpolation at node $\mathrm{n}_{\mathrm{a}}$; the analysis in this section could then be repeated for that interpolation, although this is omitted for brevity.

It is easy to see that Kim and Choi's nodal interpolation scheme is at least first order accurate in all cases, since

$$
\sum_{j}\left[q_{j \mathrm{~b}}\right]=\sum_{j}\left[\frac{\left(\frac{1}{A_{j \mathrm{~b}}}\right)}{\sum_{k}\left[\frac{1}{A_{k \mathrm{~b}}}\right]}\right]=\frac{\sum_{j}\left[\frac{1}{A_{j \mathrm{~b}}}\right]}{\sum_{k}\left[\frac{1}{A_{k \mathrm{~b}}}\right]}=1 .
$$


In general, it is not the case that $\sum_{j}\left[q_{j \mathrm{~b}}\left(\varepsilon_{j \mathrm{~b}}-\frac{1}{2} \varepsilon_{\mathrm{ab}}\right)\right]=0$ and $\sum_{j}\left[q_{j \mathrm{~b}} \delta_{j \mathrm{~b}}\right]=0$, and so Kim and Choi's nodal interpolation is only first order accurate. Figure 5 is an example of a mesh on which the interpolation at node $\mathrm{n}_{\mathrm{b}}$ is only first order accurate, although the calculations for demonstrating this are omitted for brevity.

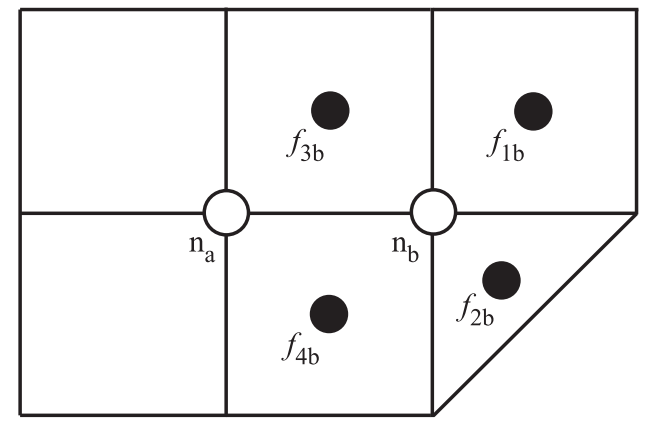

FiguRE 5. On this mesh, the interpolation at node $\mathrm{n}_{\mathrm{b}}$ is first order accurate with Kim and Choi's scheme

There is, however, one situation known to the authors where Kim and Choi's nodal scheme is second order accurate. In the case where the nodal interpolation stencil is invariant through a $180^{\circ}$ rotation (see Figure 6), every element $j$ contributing to the interpolation may be paired with a corresponding element $j^{\prime}$ directly opposite, such that $\delta_{j \mathrm{~b}}=-\delta_{j^{\prime} \mathrm{b}}$ and $\varepsilon_{j \mathrm{~b}}-\frac{1}{2} \varepsilon_{\mathrm{ab}}=-\left(\varepsilon_{j^{\prime} \mathrm{b}}-\frac{1}{2} \varepsilon_{\mathrm{ab}}\right)$. Furthermore, it is clear that $A_{j \mathrm{~b}}=A_{j^{\prime} \mathrm{b}}$, and so $q_{j \mathrm{~b}}=q_{j^{\prime} \mathrm{b}}$. It is then trivial to see that $\sum_{j}\left[q_{j \mathrm{~b}}\left(\varepsilon_{j \mathrm{~b}}-\frac{1}{2} \varepsilon_{\mathrm{ab}}\right)\right]=0$ and $\sum_{j}\left[q_{j \mathrm{~b}} \delta_{j \mathrm{~b}}\right]=0$, which means the scheme is second order accurate in this special case.

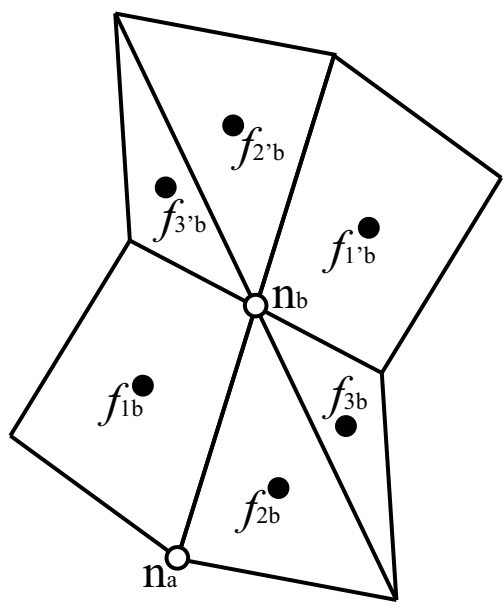

Figure 6. On this mesh, the interpolation at node $n_{b}$ is second order accurate with Kim and Choi's scheme 
3.3. Nodal interpolation in the hybrid unstructured bilinear scheme. The primary weakness of the unstructured bilinear scheme of Kim and Choi [5] is the nodal interpolation scheme, which is only first order accurate in general. This in turn degrades the accuracy of the interpolations obtained at the interpolation edge midpoint. Hence, the obvious way to improve the unstructured bilinear scheme is to replace its nodal interpolation scheme with one that is second order accurate in the general case. This had already been done by Coudiere et al. [2, although Kim and Choi did not appear to have been aware of this earlier work. The hybrid unstructured bilinear interpolation scheme presented in the present paper also uses second order accurate nodal interpolation, although unlike Coudiere et al. the scheme is used to obtain interpolations of the function at the edge midpoints as well as the gradient.

In the interior of the mesh, the hybrid unstructured bilinear scheme is simply the diamond-cell scheme, with the nodal interpolation done using moving least squares (with a first order basis polynomial). As with Kim and Choi's scheme, the stencil for the nodal interpolation by moving least squares consists of the element centroids immediately surrounding the node (although the interpolation weights are very different for the two schemes). The moving least squares method will not be elaborated here; suffice to say that in the present application it amounts to finding the plane of best fit through the function values at the surrounding element centroids, then using this plane to linearly interpolate the value of the function at the node. The reader is referred to a previous paper by the authors 1 for further information about moving least squares. In that paper, it is shown that moving least squares with an $n$th order basis polynomial is $n+1$ th order accurate in the general case, and therefore moving least squares with a first order basis polynomial is second order accurate. There are no special cases of mesh geometry known to the authors for which higher order accuracy may be obtained.

It should also be noted that in the hybrid unstructured bilinear scheme of the present paper, the interpolation for nodes and edges lying on the domain boundary was modified, so that moving least squares (with a first order basis polynomial) was used in place of Kim and Choi's [5] method. This greatly simplifies the scheme, and allows more flexible boundary conditions to be used. Since the focus of this paper is on the interpolation accuracy in the interior of the mesh, however, no more will be said about this matter.

The results of this section are summarised in Table 2

TABLE 2. Accuracy of the nodal interpolation schemes used in Kim and Choi's unstructured bilinear scheme (UBL) and this paper's hybrid unstructured bilinear scheme (HUBL). The nodal interpolation is $O\left(\Delta^{p}\right)$ accurate, with the value of $p$ given by this table.

\begin{tabular}{|l|l|l|l|}
\hline Description & Figure & In UBL & In HUBL \\
\hline General Case & 5 & $p=1$ & $p=2$ \\
$\begin{array}{l}\text { Node Stencil } \\
\text { Invariant Through } \\
180^{\circ} \text { Rotation }\end{array}$ & 6 & $p=2$ & $p=2$ \\
\hline
\end{tabular}




\section{Accuracy of the difference in interpolated nodal values}

In (2.7), it was noted that the order of accuracy of the difference between interpolated nodal values $(P)$ may be the same or greater than the order of accuracy of the individual nodal interpolations $(p)$, due to the possibility of error cancelations. While $P=p$ in the general case, there are two special cases where $P>p$, and these are explained here.

4.1. End node stencils geometrically identical through a translation. The first special case is where the stencils used for interpolating the value of the function at the end nodes are geometrically identical to each other, but of course translated by the length of the interpolation edge. In this case, the element centroids belonging to the interpolation stencil for node $\mathrm{n}_{\mathrm{a}}$ may be numbered $1 \mathrm{a}, 2 \mathrm{a}$ and so on, while the corresponding element centroids belonging to the interpolation stencil for node $\mathrm{n}_{\mathrm{b}}$ may be numbered $1 \mathrm{~b}, 2 \mathrm{~b}$, etc., as shown in Figure 7 . Further, let $f_{j \mathrm{a}}$ be the values of the function at each of the element centroids in the stencil for node $n_{a}$ and $f_{j \mathrm{~b}}$ be the values of the function at each of the corresponding element centroids in the stencil for node $\mathrm{n}_{\mathrm{b}}$. Due to the geometric identity of the stencils, the weights $q_{j}$ for corresponding element centroids in the nodal interpolations must be the same so that

$$
f_{\mathrm{a}}^{*}=\sum_{j}\left[f_{j \mathrm{a}} q_{j}\right]
$$

and

$$
f_{\mathrm{b}}^{*}=\sum_{j}\left[f_{j \mathrm{~b}} q_{j}\right]
$$

Finally, let $\delta_{j \mathrm{a}}$ and $\varepsilon_{j \mathrm{a}}$ be the coordinates of each of the element centroids in the stencil for node $\mathrm{n}_{\mathrm{a}}$ and let $\delta_{j \mathrm{~b}}$ and $\varepsilon_{j \mathrm{~b}}$ be the coordinates of each of the corresponding element centroids in the stencil for node $n_{b}$. The geometrical identity through a translation then means that

$$
\delta_{j \mathrm{~b}}-\delta_{j \mathrm{a}}=0
$$

and

$$
\varepsilon_{j \mathrm{~b}}-\varepsilon_{j \mathrm{a}}=\varepsilon_{\mathrm{ab}}
$$

The value of the function at the element centroids belonging to node $n_{a}$ can then be represented with a Taylor series as

$$
\begin{aligned}
f_{j \mathrm{a}}= & f_{\mathrm{m}}+\varepsilon_{j \mathrm{a}} \frac{\partial f_{\mathrm{m}}}{\partial \varepsilon}+\delta_{j \mathrm{a}} \frac{\partial f_{\mathrm{m}}}{\partial \delta}+\frac{\varepsilon_{j \mathrm{a}}^{2}}{2 !} \frac{\partial^{2} f_{\mathrm{m}}}{\partial \varepsilon^{2}} \\
& +\frac{2 \varepsilon_{j \mathrm{a}} \delta_{j \mathrm{a}}}{2 !} \frac{\partial^{2} f_{\mathrm{m}}}{\partial \varepsilon \partial \delta}+\frac{\delta_{j \mathrm{a}}^{2}}{2 !} \frac{\partial^{2} f_{\mathrm{m}}}{\partial \delta^{2}}+O\left(\Delta^{3}\right)
\end{aligned}
$$




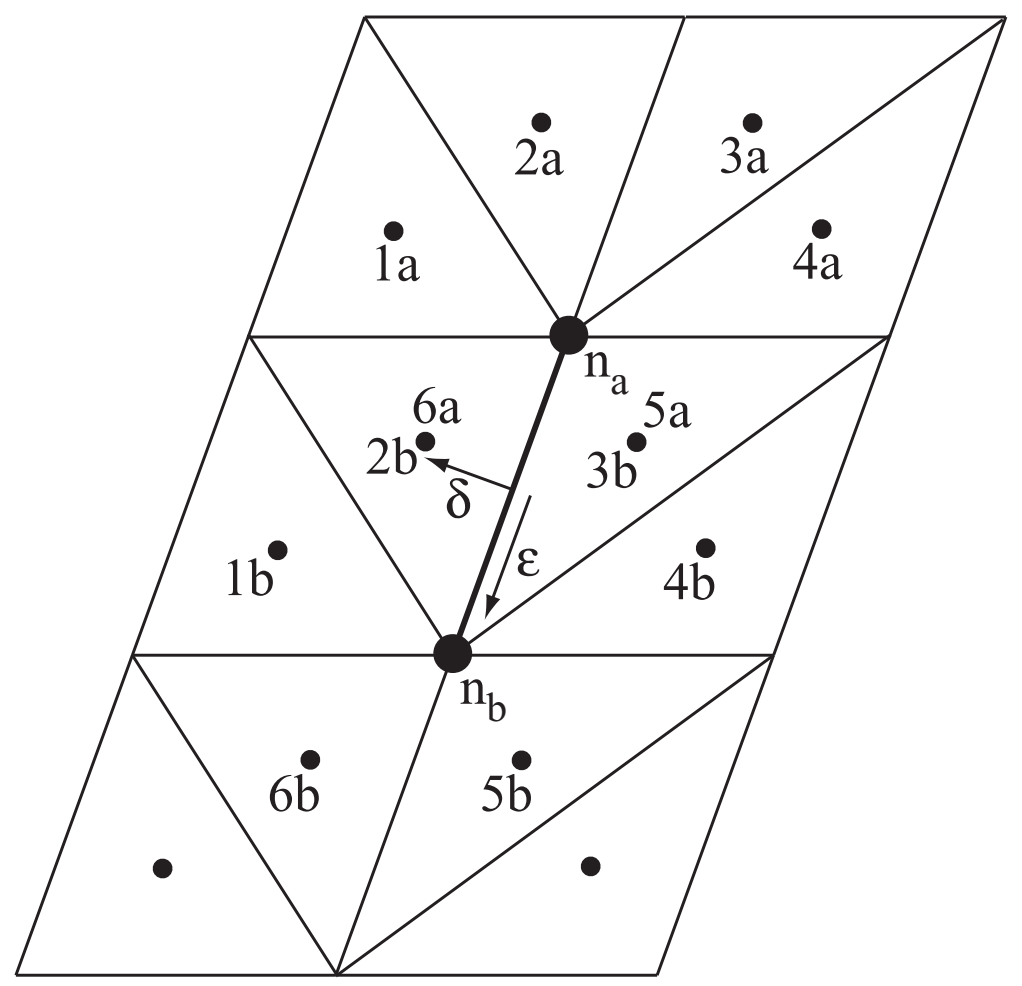

FiguRE 7. An interpolation edge whose end node stencils are geometrically identical through a translation.

with a similar expression for $f_{j \mathrm{~b}}$. Substituting these expressions into the difference between (4.2) and (4.1) and then subtracting (2.4) yields

$$
\begin{aligned}
\left(f_{\mathrm{b}}^{*}\right. & \left.-f_{\mathrm{a}}^{*}\right)-\left(f_{\mathrm{b}}-f_{\mathrm{a}}\right)=\frac{\partial f_{\mathrm{m}}}{\partial \varepsilon}\left\{\sum_{j}\left[q_{j}\left(\varepsilon_{j \mathrm{~b}}-\varepsilon_{j \mathrm{a}}\right)\right]-\varepsilon_{\mathrm{ab}}\right\} \\
& +\frac{\partial f_{\mathrm{m}}}{\partial \delta} \sum_{j}\left[q_{j}\left(\delta_{j \mathrm{~b}}-\delta_{j \mathrm{a}}\right)\right] \\
& +\frac{1}{2 !} \cdot \frac{\partial^{2} f_{\mathrm{m}}}{\partial \varepsilon^{2}} \sum_{j}\left[q_{j}\left(\varepsilon_{j \mathrm{~b}}^{2}-\varepsilon_{j \mathrm{a}}^{2}\right)\right]+\frac{2}{2 !} \cdot \frac{\partial^{2} f_{\mathrm{m}}}{\partial \varepsilon \partial \delta} \sum_{j}\left[q_{j}\left(\varepsilon_{j \mathrm{~b}} \delta_{j \mathrm{~b}}-\varepsilon_{j \mathrm{a}} \delta_{j \mathrm{a}}\right)\right] \\
& +\frac{1}{2 !} \cdot \frac{\partial^{2} f_{\mathrm{m}}}{\partial \delta^{2}} \sum_{j}\left[q_{j}\left(\delta_{j \mathrm{~b}}^{2}-\delta_{j \mathrm{a}}^{2}\right)\right]+O\left(\Delta^{3}\right) .
\end{aligned}
$$


Equation (4.6) must be simplified using (4.3) and (4.4) along with the identity $\varepsilon_{j \mathrm{~b}}+\varepsilon_{j \mathrm{a}} \equiv\left(\varepsilon_{j \mathrm{a}}+\frac{1}{2} \varepsilon_{\mathrm{ab}}\right)+\left(\varepsilon_{j \mathrm{~b}}-\frac{1}{2} \varepsilon_{\mathrm{ab}}\right)$. This yields

$$
\begin{aligned}
\left(f_{\mathrm{b}}^{*}-f_{\mathrm{a}}^{*}\right)-\left(f_{\mathrm{b}}-f_{\mathrm{a}}\right)= & \varepsilon_{\mathrm{ab}} \frac{\partial f_{\mathrm{m}}}{\partial \varepsilon}\left\{\sum_{j}\left[q_{j}\right]-1\right\} \\
& +\frac{\varepsilon_{\mathrm{ab}}}{2 !} \cdot \frac{\partial^{2} f_{\mathrm{m}}}{\partial \varepsilon^{2}} \sum_{j}\left[q_{j}\left(\varepsilon_{j \mathrm{a}}+\frac{1}{2} \varepsilon_{\mathrm{ab}}\right)\right] \\
& +\frac{\varepsilon_{\mathrm{ab}}}{2 !} \cdot \frac{\partial^{2} f_{\mathrm{m}}}{\partial \varepsilon^{2}} \sum_{j}\left[q_{j}\left(\varepsilon_{j \mathrm{~b}}-\frac{1}{2} \varepsilon_{\mathrm{ab}}\right)\right] \\
& +\frac{2 \varepsilon_{\mathrm{ab}}}{2 !} \cdot \frac{\partial^{2} f_{\mathrm{m}}}{\partial \varepsilon \partial \delta} \sum_{j}\left[q_{j} \delta_{j \mathrm{a}}\right]+O\left(\Delta^{3}\right) .
\end{aligned}
$$

If the nodal interpolation is second order accurate, then

$$
\sum_{j}\left[q_{j}\right]=1, \sum_{j}\left[q_{j}\left(\varepsilon_{j \mathrm{a}}+\frac{1}{2} \varepsilon_{\mathrm{ab}}\right)\right]=0, \sum_{j}\left[q_{j}\left(\varepsilon_{j \mathrm{~b}}-\frac{1}{2} \varepsilon_{\mathrm{ab}}\right)\right]=0 \text { and } \sum_{j}\left[q_{j} \delta_{j \mathrm{a}}\right]=0,
$$

and so the error in the interpolated nodal difference is third order. On the other hand, if the nodal interpolation is first order accurate, then in general it is only true that $\sum_{j}\left[q_{j}\right]=1$ and so the error in the interpolated nodal difference is second order. These results are summarised in Table 3

4.2. End node stencils geometrically identical through a translation and $180^{\circ}$ rotation. The second special case is where the stencils used for interpolating the value of the function at the end nodes are geometrically identical to each other, but this time when translated by the length of the interpolation edge and rotated by $180^{\circ}$. Once again, the element centroids belonging to the interpolation stencil for node $\mathrm{n}_{\mathrm{a}}$ may be numbered $1 \mathrm{a}, 2 \mathrm{a}$ and so on, while the corresponding element centroids belonging to the interpolation stencil for node $n_{b}$ may be numbered $1 b$, $2 \mathrm{~b}$, etc., as shown in Figure 8 . Further, let $f_{j \mathrm{a}}$ be the values of the function at each of the element centroids in the stencil for node $n_{\mathrm{a}}$ and $f_{j \mathrm{~b}}$ be the values of the function at each of the corresponding element centroids in the stencil for node $\mathrm{n}_{\mathrm{b}}$. Due to the geometric identity of the stencils, the weights $q_{j}$ for corresponding element centroids in the nodal interpolations must be the same, so that (4.1) and (4.2) also apply in this context.

Finally, let $\delta_{j a}$ and $\varepsilon_{j a}$ be the coordinates of each of the element centroids in the stencil for node $\mathrm{n}_{\mathrm{a}}$, and let $\delta_{j \mathrm{~b}}$ and $\varepsilon_{j \mathrm{~b}}$ be the coordinates of each of the corresponding element centroids in the stencil for node $n_{b}$. The geometrical identity through a translation and $180^{\circ}$ rotation then means that

$$
\delta_{j \mathrm{~b}}+\delta_{j \mathrm{a}}=0
$$

and

$$
\varepsilon_{j \mathrm{~b}}+\varepsilon_{j \mathrm{a}}=0
$$




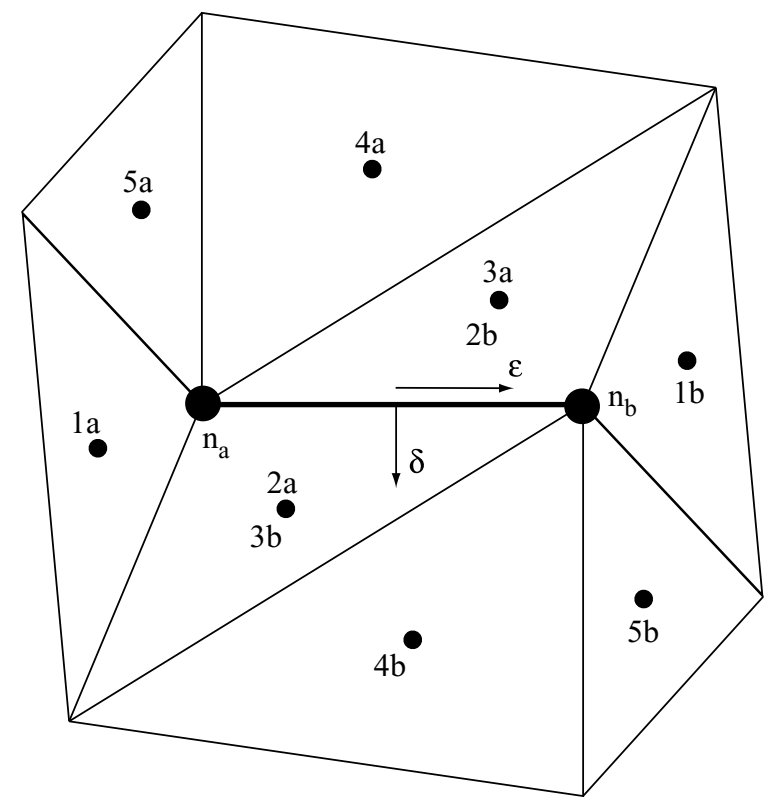

FiguRE 8. An interpolation edge whose end node stencils are geometrically identical through a translation and $180^{\circ}$ rotation.

Equation (4.6) applies to this special case also, but in this situation it must be simplified using (4.8) and (4.9) along with the identity

$$
\varepsilon_{j \mathrm{~b}}-\varepsilon_{j \mathrm{a}} \equiv\left(\varepsilon_{j \mathrm{~b}}-\frac{1}{2} \varepsilon_{\mathrm{ab}}\right)-\left(\varepsilon_{j \mathrm{a}}+\frac{1}{2} \varepsilon_{\mathrm{ab}}\right)+\varepsilon_{\mathrm{ab}}
$$

This yields

$$
\begin{aligned}
\left(f_{\mathrm{b}}^{*}\right. & \left.-f_{\mathrm{a}}^{*}\right)-\left(f_{\mathrm{b}}-f_{\mathrm{a}}\right)=\varepsilon_{\mathrm{ab}} \frac{\partial f_{\mathrm{m}}}{\partial \varepsilon}\left(\sum_{j}\left[q_{j}\right]-1\right) \\
& +\frac{\partial f_{\mathrm{m}}}{\partial \varepsilon} \sum_{j}\left[q_{j}\left(\varepsilon_{j \mathrm{~b}}-\frac{1}{2} \varepsilon_{\mathrm{ab}}\right)\right] \\
& -\frac{\partial f_{\mathrm{m}}}{\partial \varepsilon} \sum_{j}\left[q_{j}\left(\varepsilon_{j \mathrm{a}}+\frac{1}{2} \varepsilon_{\mathrm{ab}}\right)\right]+2 \frac{\partial f_{\mathrm{m}}}{\partial \delta} \sum_{j}\left[q_{j} \delta_{j \mathrm{~b}}\right]+O\left(\Delta^{3}\right) .
\end{aligned}
$$

If the nodal interpolation is second order accurate, then

$$
\sum_{j}\left[q_{j}\right]=1, \sum_{j}\left[q_{j}\left(\varepsilon_{j \mathrm{~b}}-\frac{1}{2} \varepsilon_{\mathrm{ab}}\right)\right]=0, \sum_{j}\left[q_{j}\left(\varepsilon_{j \mathrm{a}}+\frac{1}{2} \varepsilon_{\mathrm{ab}}\right)\right]=0 \text { and } \sum_{j}\left[q_{j} \delta_{j \mathrm{~b}}\right]=0
$$

and so the error in the interpolated nodal difference is third order. On the other hand, if the nodal interpolation is first order accurate, then in general it is only true that $\sum_{j}\left[q_{j}\right]=1$ and so the error in the interpolated nodal difference is first order. These results are summarised in Table 3. 
TABle 3. Dependency of the interpolated nodal difference on the nodal interpolation scheme and mesh geometry. The nodal interpolation is $O\left(\Delta^{p}\right)$ accurate, while the interpolated nodal difference is $O\left(\Delta^{P}\right)$ accurate.

\begin{tabular}{|c|c|c|c|}
\hline Description & Figure & If $p=1$ & If $p=2$ \\
\hline General Case & - & $P=1$ & $P=2$ \\
\hline $\begin{array}{l}\text { End Node Stencils } \\
\text { Geometrically Identical } \\
\text { Through Translation }\end{array}$ & 7 & $P=2$ & $P=3$ \\
\hline $\begin{array}{l}\text { End Node Stencils } \\
\text { Geometrically Identical } \\
\text { Through Translation } \\
\text { and } 180^{\circ} \text { Rotation }\end{array}$ & 8 & $P=1$ & $P=3$ \\
\hline
\end{tabular}

\section{Summary}

In view of the complexity of the preceding theory, Figure 9 is provided as a quick guide to determining the order of accuracy that applies in any situation covered by this paper. This master flowchart encapsulates the logic necessary for using the results from Tables 2 and 3 . Note that different orders of accuracy may be obtained for the function interpolation and the two components of the gradient. The orders of accuracy that are obtained depend on the local mesh geometry near the interpolation edge, as well as the scheme chosen for the nodal interpolation. Figure 9 allows the order of accuracy of the nodal difference $(P)$ to be determined for a given situation, and having obtained the value of $P$ it is then necessary to consult the relevant flowchart from Figures 2 to 4 to find the orders of accuracy for the function and gradient interpolation at the edge midpoint.

In order to illustrate the theory, Figure 10 provides an example mesh for each of the special cases identified. For most of the examples, only the highlighted edge belongs to the nominated special case. Note that special cases E to I are only possible when using unstructured bilinear interpolation, while case B is only possible with hybrid unstructured bilinear interpolation.

At this point, it should be mentioned that the mesh used by Kim and Choi [5] for an empirical demonstration that their scheme is "second order accurate" was of the same form as case D of Figure 10. As is noted, the vertical edges of this mesh belong to case $\mathrm{D}$, and so the function interpolations on these edges are second order accurate while the spatial derivatives are first order accurate. The horizontal edges of the mesh belong to case D also, while the diagonal edges belong to case A (with second order accuracy for the function interpolation and both spatial derivatives). Across the entire mesh, therefore, the spatial derivatives vary between first and second order accuracy. More importantly, the function interpolation across the entire mesh is second order accurate, which explains why Kim and Choi were able to get second order convergence for their finite volume Navier-Stokes simulations on this mesh, even though the unstructured bilinear scheme that they used is only first order accurate in general. 


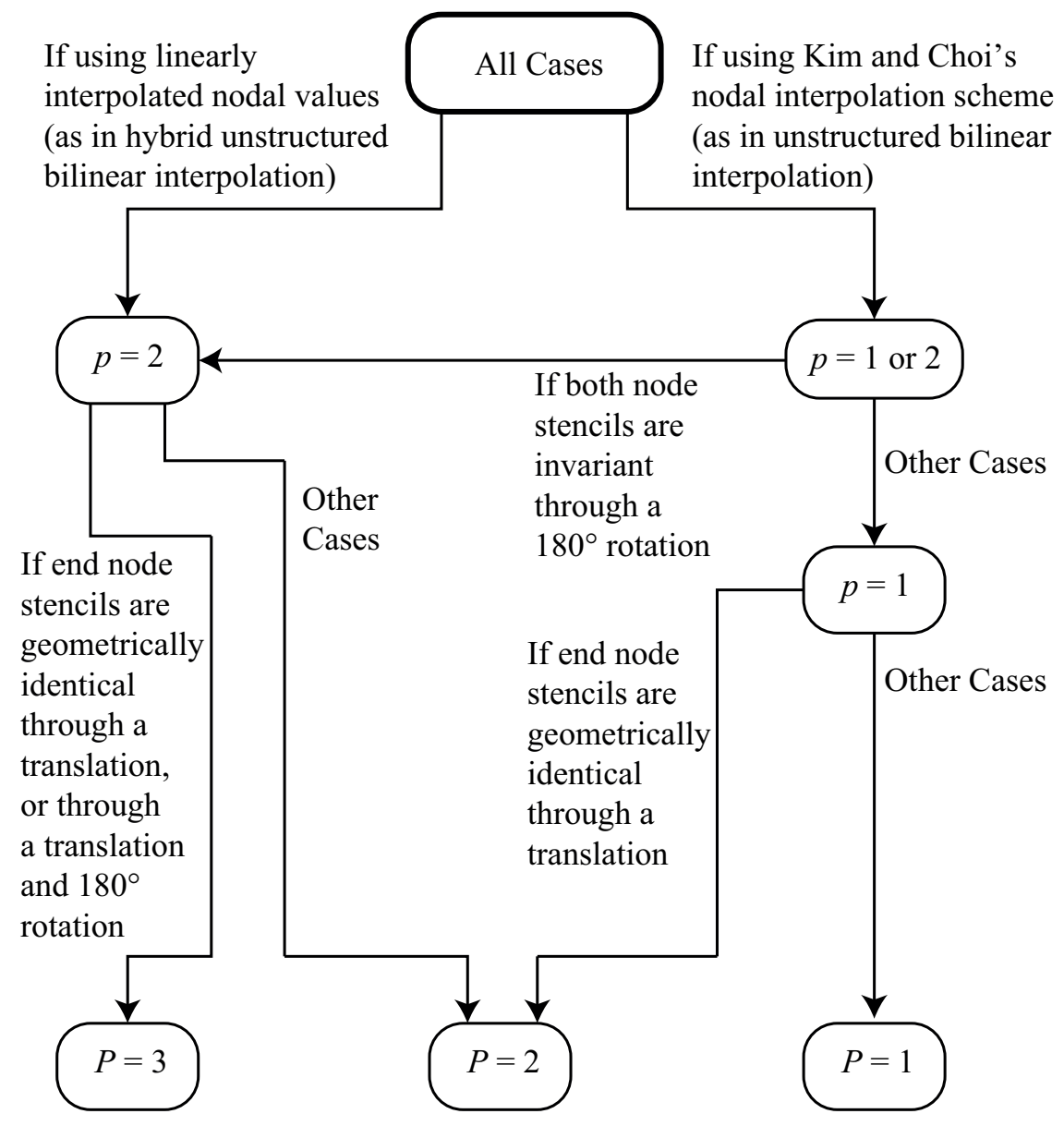

Figure 9. Master flowchart for determining accuracy. The leaves $P=3, P=2$ and $P=1$ link to the trees in Figures 2, 3] and 4, respectively. 


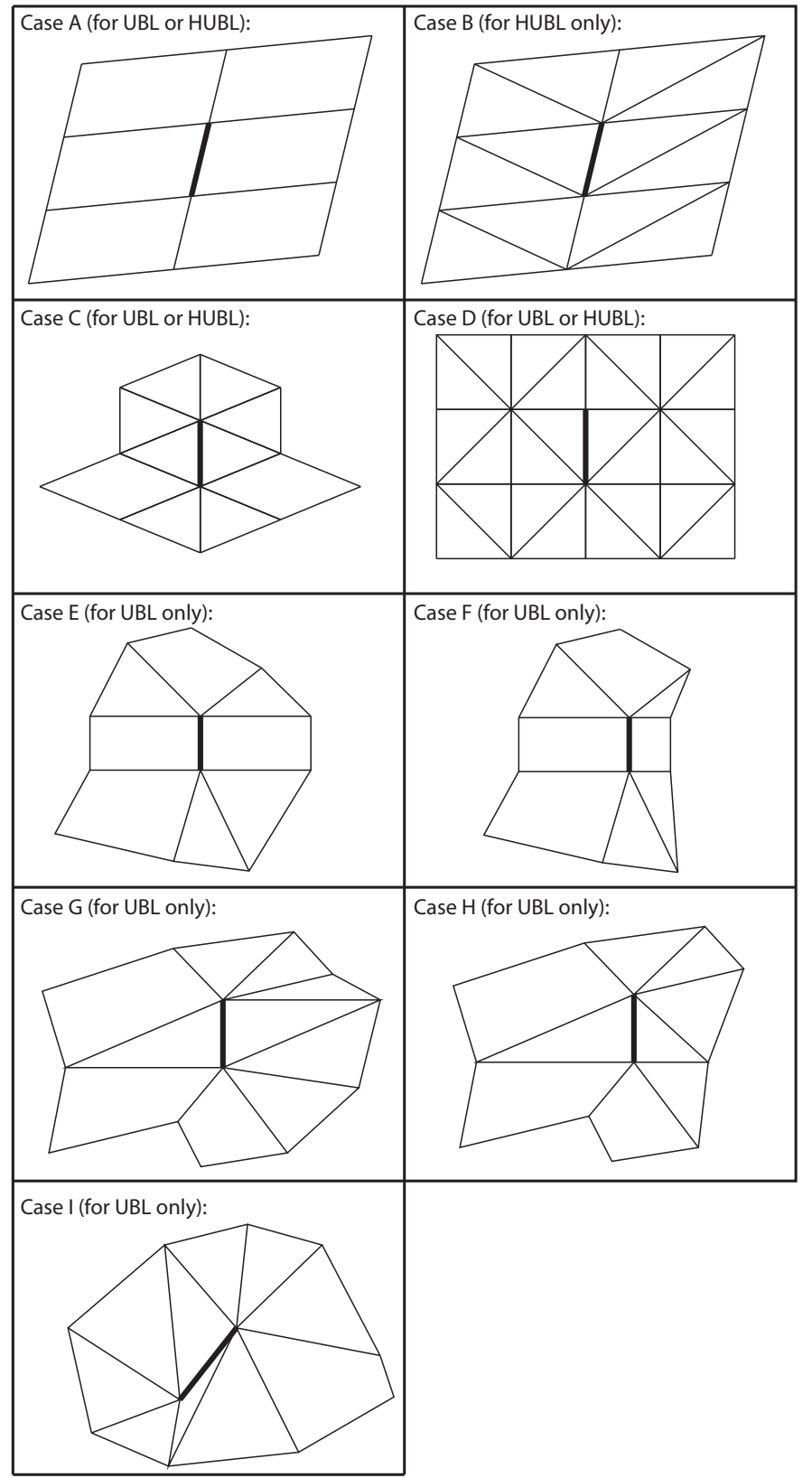

FiguRE 10. Examples of the special cases for interpolation using the unstructured bilinear (UBL) or hybrid unstructured bilinear (HUBL) schemes. Case D is the general case for HUBL, while case I is the general case for UBL. 


\section{Results}

6.1. Empirical metrics for determining order of accuracy. It is useful to study the order of accuracy of interpolation schemes by empirical means, in order to provide additional evidence in support of the theoretical framework. To this end, test function analysis may be used along similar lines to that used by [8] and [1. This analysis tests the accuracy of an interpolation scheme on a given mesh by taking some analytical function $f$ and evaluating this test function at the element centroids (and boundary edge midpoints) of the mesh. These values may be used as inputs to the interpolation scheme, from which estimates of the value of $f$ (and its spatial derivatives) at the edge midpoints may be obtained. Finally, the accuracy of the interpolation scheme may be assessed by comparing the interpolated values at the edge midpoints with values obtained from the analytical function.

Specifically, the overall $f$ interpolation accuracy may be gauged by computing

$$
E_{f}=\frac{\sqrt{\sum_{i \in \text { edges }}\left(f_{i}^{*}-f_{i}\right)^{2}}}{\left(f_{\max }-f_{\min }\right) \sqrt{n_{\text {edges }}}},
$$

where $f_{i}^{*}$ is the interpolated value of $f$ on edge $i, f_{i}$ is the analytical value of $f$ on edge $i, n_{\text {edges }}$ is the number of edges used to compute the statistic and $f_{\max }$ and $f_{\min }$ represent the extreme values taken by $f$ over the domain. This quantity is the root mean square interpolation error in $f$, normalised by the range of the test function. This metric may be computed over all the edges in the mesh, or else over some specific subset of these; the set of edges included will be noted where this metric is used. Likewise, the error in the interpolation of the $\delta$ direction spatial derivative may be gauged using

$$
E_{d f / d \delta}=\frac{\sqrt{\sum_{i \in \text { edges }}\left(\left.\frac{d f *}{d \delta}\right|_{i}-\left.\frac{d f}{d \delta}\right|_{i}\right)^{2}}}{\left(\left.\frac{d f}{d \delta}\right|_{\max }-\left.\frac{d f}{d \delta}\right|_{\min }\right) \sqrt{n_{\text {edges }}}}
$$

while the error in the interpolation of the $\varepsilon$ direction spatial derivative may be gauged using $E_{d f / d \varepsilon}$, which is given by a similar expression.

An appropriate choice for $f$ is a sinusoidal function in both $x$ and $y$, since this is an infinite order function, varies with both $x$ and $y$ and has the same smoothness properties throughout the domain. Such functions were used by [8] and [1] to conduct similar tests. Most importantly, a sinusoidal function has a convenient parameter (wavelength), which may be varied in order to alter the smoothness of the function in comparison to the mesh. Experience and common sense suggests that test function values will be easier to replicate accurately using interpolation if the test function is smooth at the scale of the mesh elements. For the analytical values of a sinusoidal test function to be reproduced accurately by interpolation, there needs to be several samples per period of the wave. In other words, the wavelength of a sinusoidal test function needs to be several times the size of a typical mesh element if accurate reproduction is to be obtained. The number of mesh elements $N$ per test function period may then be taken as a measure of the difficulty of the test function. The performance of a given interpolation scheme may be gauged by using a range of values of $N$, in order to find out how quickly the scheme's error decays as $N$ is increased. Specifically, a graph of $\log _{10}(E)$ versus 
$\log _{10}(N)$ may be shown to have a gradient equal to the negative of the order of accuracy.

Consider a regular rectangular mesh with a mesh spacing of $\Delta_{x}$ in the $x$ direction and $\Delta_{y}$ in the $y$ direction. In view of the previous discussion, a suitable test function for this class of mesh is

$$
f(x, y)=\cos \left(\frac{2 \pi}{N \Delta_{x}} x+\frac{2 \pi}{N \Delta_{y}} y\right),
$$

where $N$ is a parameter which determines the number of data points per period of the wave along each coordinate axis. $N$ does not need to be an integer, but since $N=2$ corresponds to the Nyquist limit, values of $N$ greater than 2 must be selected. The upper bound on $N$ is determined by the need to include at least a full period of the wave inside the domain, so that the full range of possible $f$ values and spatial derivatives are available from the test function at the sample points. Specifically, the maximum value of $N$ is selected so that:

- The range of $f$ as sampled over the domain is at least $99 \%$ of the range of $f$ over the entire $(x, y)$ plane.

- The range of $\frac{d f}{d x}$ as sampled over the domain is at least $99 \%$ of the range of $\frac{d f}{d x}$ over the entire $(x, y)$ plane.

- The range of $\frac{d f}{d y}$ as sampled over the domain is at least $99 \%$ of the range of $\frac{d f}{d y}$ over the entire $(x, y)$ plane.

The test function may be extended to irregular meshes if some rough approximations are applied. To this end, a general mesh will be represented by a regular rectangular mesh, which is in some sense equivalent to the original mesh. Let element $i$ have a maximum $x$ value (i.e., at its rightmost corner node) of $x_{i \text {,max }}$, minimum $x$ value of $x_{i, \min }$, maximum $y$ value of $y_{i, \max }$ and minimum $y$ value of $y_{i, \min }$. Further, let $A_{i}$ be the area of element $i$ and $M$ be the number of elements in the mesh. The aspect ratio of the rectangular elements in the equivalent mesh should be similar to the aspect ratio of the elements in the original mesh, and so an equivalent aspect ratio $R$ may be defined as

$$
R=\frac{\frac{1}{M} \sum_{i}\left(y_{i, \max }-y_{i, \min }\right)}{\frac{1}{M} \sum_{i}\left(x_{i, \max }-x_{i, \min }\right)}=\frac{\sum_{i}\left(y_{i, \max }-y_{i, \min }\right)}{\sum_{i}\left(x_{i, \max }-x_{i, \min }\right)} .
$$

In effect, $R$ is the ratio between the average $y$ range of the elements to the average $x$ range. This measure gives reasonable results on a range of meshes. Next, a basic mesh scale $\Delta$ may be calculated by taking the square root of the average element area, i.e.,

$$
\Delta=\sqrt{\frac{1}{M} \sum_{i} A_{i}}
$$

Finally, the equivalent mesh scales in the $x$ and $y$ directions may be computed using

$$
\Delta_{x}=\frac{\Delta}{\sqrt{R}}
$$

and

$$
\Delta_{y}=\Delta \sqrt{R}
$$


so that $\Delta_{x} \Delta_{y}=\frac{1}{M} \sum_{i} A_{i}$ and $\frac{\Delta_{y}}{\Delta_{x}}=R$. Note that if this method is applied to a regular rectangular mesh, the equivalent mesh is the same as the original mesh, as expected. With this approximation, test function (6.3) may be applied to unstructured meshes of triangles and/or quadrilaterals, producing consistent results.

6.2. Accuracy of the schemes on a typical unstructured mesh. In order to assess the typical accuracy of the unstructured bilinear scheme, the unstructured mesh of irregular quadrilaterals shown in Figure 11 was used, and plots of the root mean square errors (Figure 12) were obtained. All edges of the mesh were included. The following may be observed:

- The curve for $E_{d f / d \varepsilon}$ is fairly flat, which is exactly what is expected for the general case (I) with zeroth order accuracy for the $\varepsilon$ derivative.

- The curve for $E_{d f / d \delta}$ has a gradient of nearly -2 at very small values of $N$, which indicates nearly second order accuracy when the mesh is very large scale. This can be explained by the fact that there are many edges in the regular rectangular areas of this mesh which belong to case A. In addition, there are other edges which "almost" belong to case A, in the sense that a very slight modification to the mesh geometry would make them belong. The second order convergence rate of the errors on such edges at first gives the illusion of second order accuracy overall.

- However, as $N$ increases, the gradient of the $E_{d f / d \delta}$ curve quickly reduces to -1 and then to zero. This is because the initially rapid decrease in the errors for the edges that belong to class A causes them to dwindle into insignificance as $N$ increases further, so that the root mean square error becomes dominated by edges for which $E_{d f / d \delta}$ has a lower order of accuracy. Eventually, the errors associated with edges having zeroth order accuracy for $E_{d f / d \delta}$ make up the vast majority of the root mean square error, and so the error curve flattens out to zero gradient.

- The $E_{f}$ curve has a gradient of -2 for a wide range of scales, indicating second order accuracy. This is consistent with the theory, as $\varepsilon_{\mathrm{m}}$ is very close to zero for most edges of the mesh, and so the second order error term of (2.21) dominates the first order error term at all but the smallest mesh scales. (There is evidence of the presence of a very small first order error term in the slight decrease in the convergence rate at very small mesh scales.)

The numerical experiment suggests that the unstructured bilinear scheme may be relied on for second order $f$ interpolation accuracy, providing that the mesh chosen has similar properties to that studied. This is very useful, as this mesh was chosen as a simple, practical mesh, with no special mesh design principles applied. The $\varepsilon$ derivative is zeroth order accurate, and should not be relied on for anything but the roughest of calculations. The $\delta$ derivative, on the other hand, has much better accuracy. This is important, as in many finite volume computations (such as the convection-diffusion equation and the incompressible Navier-Stokes equations) it is the only spatial derivative which is actually required (even though the formulation may be expressed in terms of $x$ and $y$ spatial derivatives). Thus unstructured bilinear interpolation is a workable scheme for use in finite volume solvers requiring only the interpolation of $f$ and $\frac{\partial f}{\partial \delta}$ on the mesh edges, as long as meshes with 
similar properties to Figure 11 are used. This explains the success of the numerical simulations of Kim and Choi [5] on a variety of irregular, unstructured meshes.

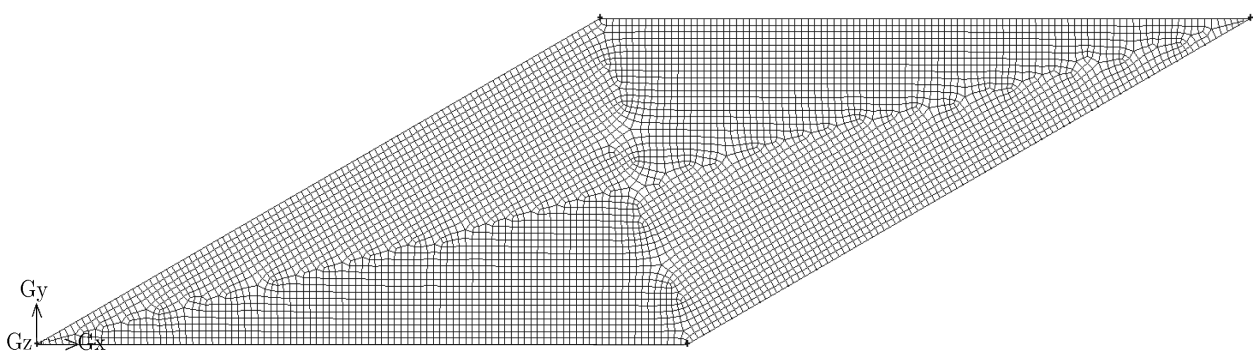

FIGURE 11. An irregular mesh of quadrilaterals.

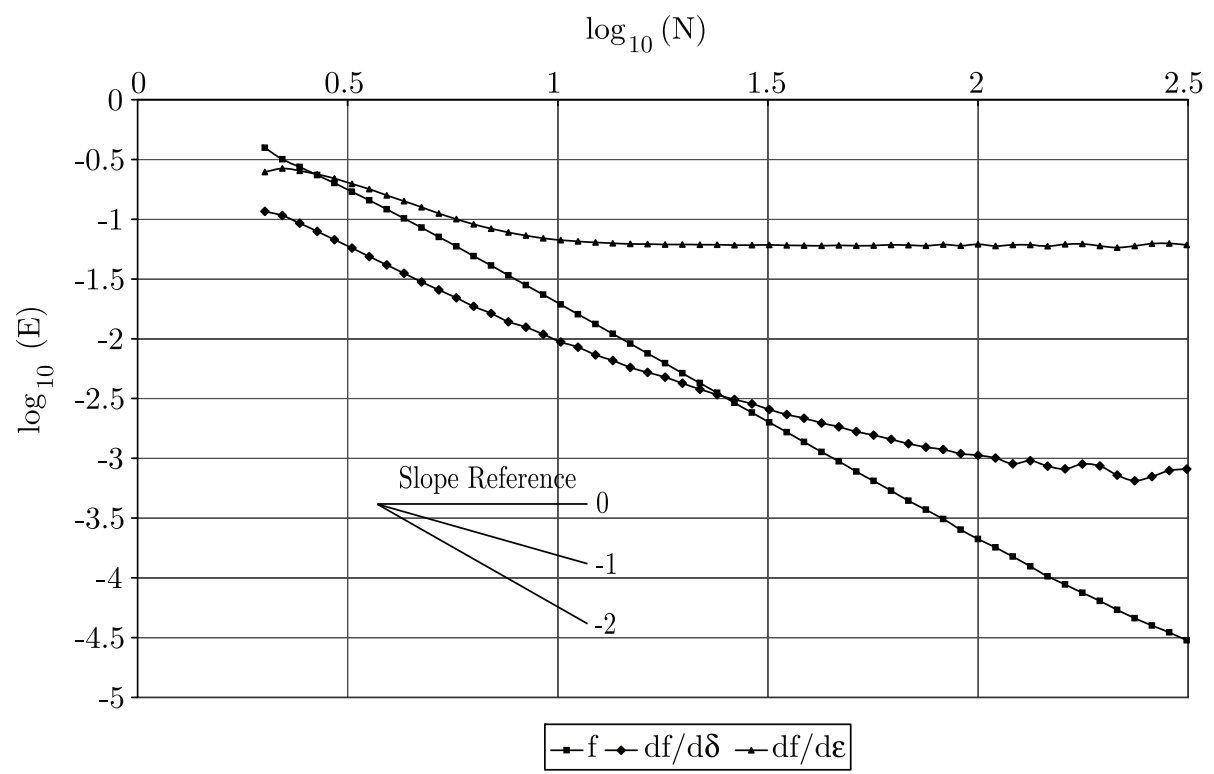

FiguRE 12. Unstructured bilinear interpolation test results on an irregular mesh of quadrilaterals. Plots of $E_{f}, E_{d f / d \delta}$ and $E_{d f / d \varepsilon}$ are shown. All edges are included in the statistics.

In order to assess the typical accuracy of the hybrid unstructured bilinear scheme, the unstructured mesh of irregular quadrilaterals shown in Figure 11 was used, and plots of the root mean square errors (Figure 13) were obtained. All edges of the mesh were included. The following may be observed:

- The curve for $E_{d f / d \varepsilon}$ has a gradient of about -2 at very small values of $N$, which indicates nearly second order accuracy when the mesh is very large scale. This is due to the presence of many case A (or almost case A) edges in the mesh, although it is not clear why these have such a strong effect on $E_{d f / d \varepsilon}$ in comparison to when unstructured bilinear interpolation was used. However, at larger values of $N$ the error becomes dominated by those edges 
with a first order convergence rate for $E_{d f / d \varepsilon}$, as expected for the general case (D).

- The curve for $E_{d f / d \delta}$ has a gradient of about -1 , indicating first order accuracy as expected for the general case (D).

- The $E_{f}$ curve has a gradient of about -2 over a wide range of scales, indicating second order accuracy as expected for the general case (D).

The numerical experiment suggests that the hybrid unstructured bilinear scheme may be relied on for second order $f$ interpolation accuracy and first order accurate spatial derivative interpolation, when using typical meshes such as this. Note in particular that the accuracy of the $\varepsilon$ direction spatial derivative is greatly improved, in comparison to the conventional unstructured bilinear scheme.

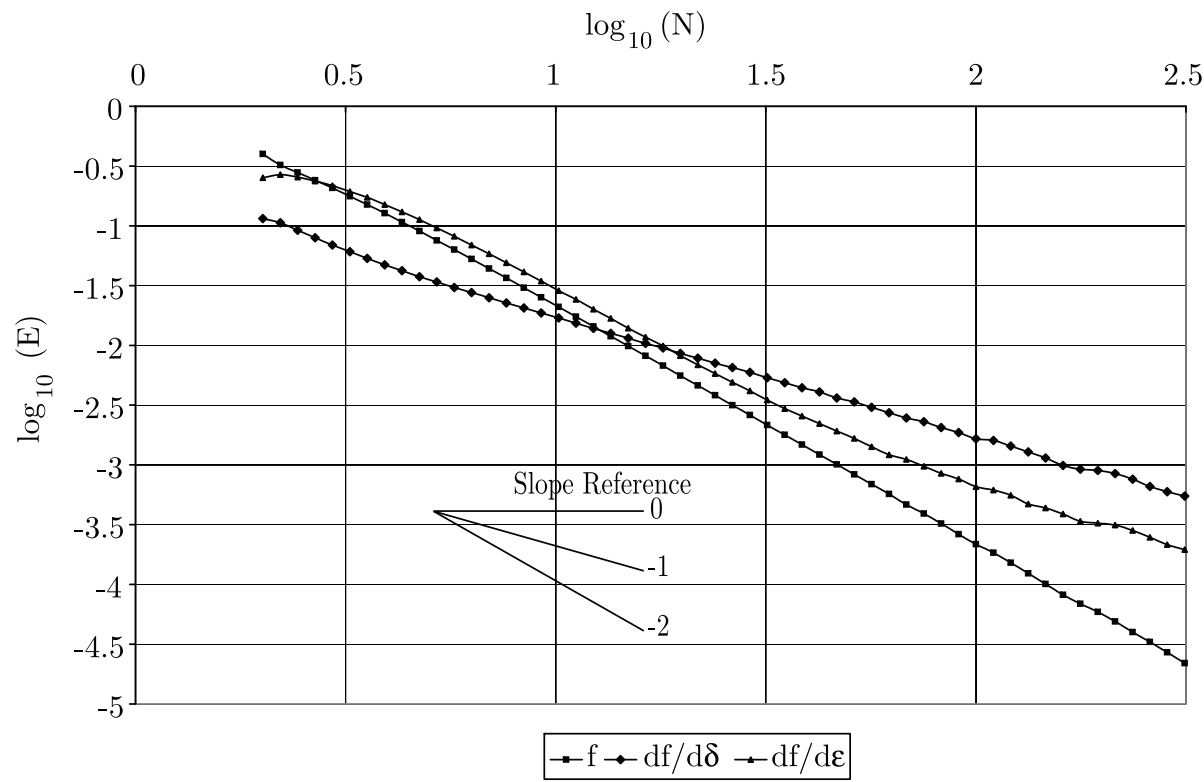

FIGURE 13. Hybrid unstructured bilinear interpolation test results on an irregular mesh of quadrilaterals. Plots of $E_{f}, E_{d f / d \delta}$ and $E_{d f / d \varepsilon}$ are shown. All edges are included in the statistics.

6.3. Demonstrating the improvement from the hybrid unstructured bilinear scheme. The deficiencies of the unstructured bilinear scheme are difficult to observe on many meshes, owing to the fact that for most edges, the line joining the two adjacent element centroids intersects the interpolation edge very close to its midpoint. This means the magnitude of $\varepsilon_{\mathrm{m}}$ is small in comparison to the mesh scale, and so the interpolated value of $f$ at the edge midpoint is approximately second order accurate. On a mesh of the same form as in Figure 14, however, the vertical edges have values of $\varepsilon_{\mathrm{m}}$ which are much more significant, especially for the short vertical edges. This allows the first order accuracy of the unstructured bilinear scheme to be observed, which is what the theory predicts for the general case. In order for the effect to be isolated, the error statistics are calculated using only the vertical edges of the mesh. Edges with an end node on the domain boundary 


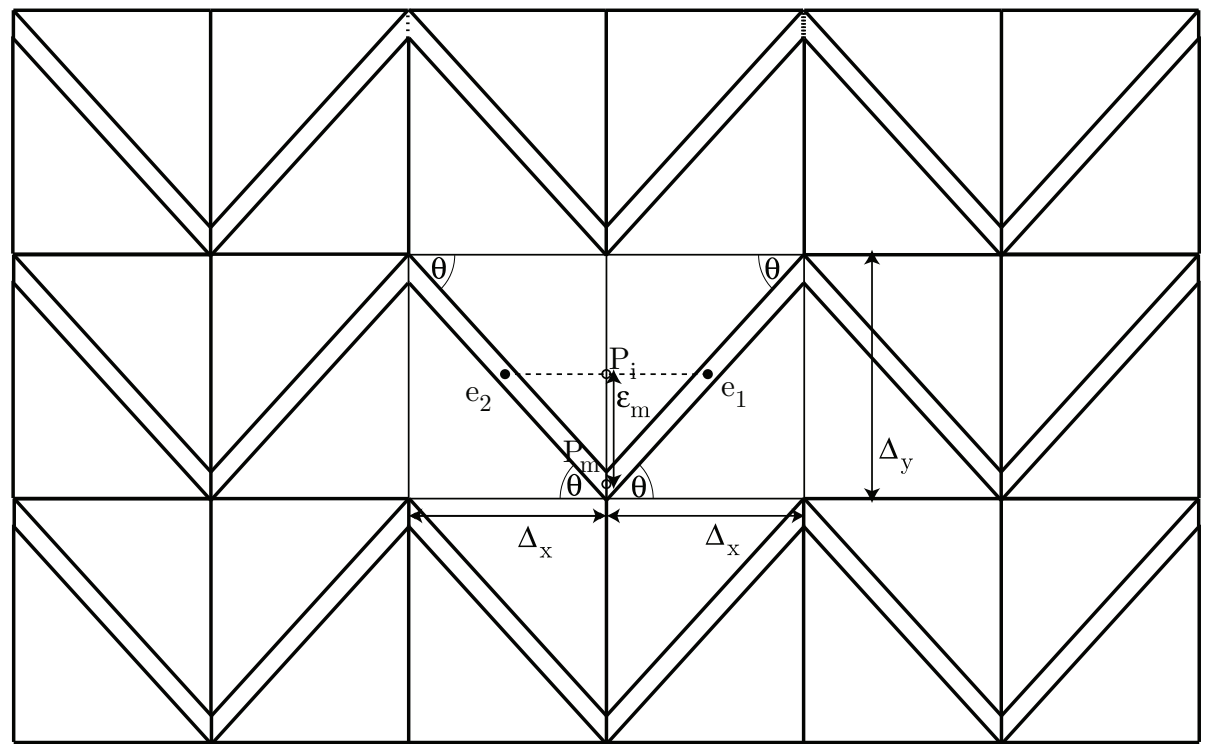

FiguRE 14. Schematic of the "chevron" mesh, demonstrating the first order accuracy of the unstructured bilinear scheme. First order errors dominate on the edge with its midpoint marked $P_{m}$, as well as on the equivalent edges elsewhere in the mesh.

are also excluded, limiting the results to the behaviour of the internal scheme. It should be noted that the edges for which the error statistics are computed belong to case $\mathrm{H}$. The mesh was $1 \mathrm{~m} \times 1 \mathrm{~m}$ square, with $\Delta_{x}=\Delta_{y}=0.02 \mathrm{~m}$ and $\theta=40^{\circ}$. Choosing a $\theta$ value so close to $45^{\circ}$ makes the $\varepsilon_{\mathrm{m}}$ value for the short vertical edges quite large, in comparison to the local mesh scale. This ensures that the first order error term dominates the second order term at all mesh scales. The test function results are as shown in Figure 15. It can be seen that:

- The $E_{f}$ curve has a gradient of approximately -1 over a wide range of scales, indicating first order accuracy. This is consistent with the theoretical prediction for case $\mathrm{H}$ edges.

- The $E_{d f / d \varepsilon}$ curve is fairly flat, indicating zero order accuracy. This is consistent with the theoretical prediction for case $\mathrm{H}$ edges.

- The $E_{d f / d \delta}$ curve has a gradient of -1 over a wide range of scales, indicating first order accuracy. This is consistent with the theoretical prediction for case $\mathrm{H}$ edges.

- At very small values of $N$, all three curves depart slightly from the expected straight line relationship. This is difficult to explain, but minor.

This test case is of critical importance, as it provides strong empirical evidence to support the theoretical claim that the unstructured bilinear scheme is only first order accurate in general, in contrast to the claims of Kim and Choi [5]. It also supports the existence of the special case $\mathrm{H}$. 


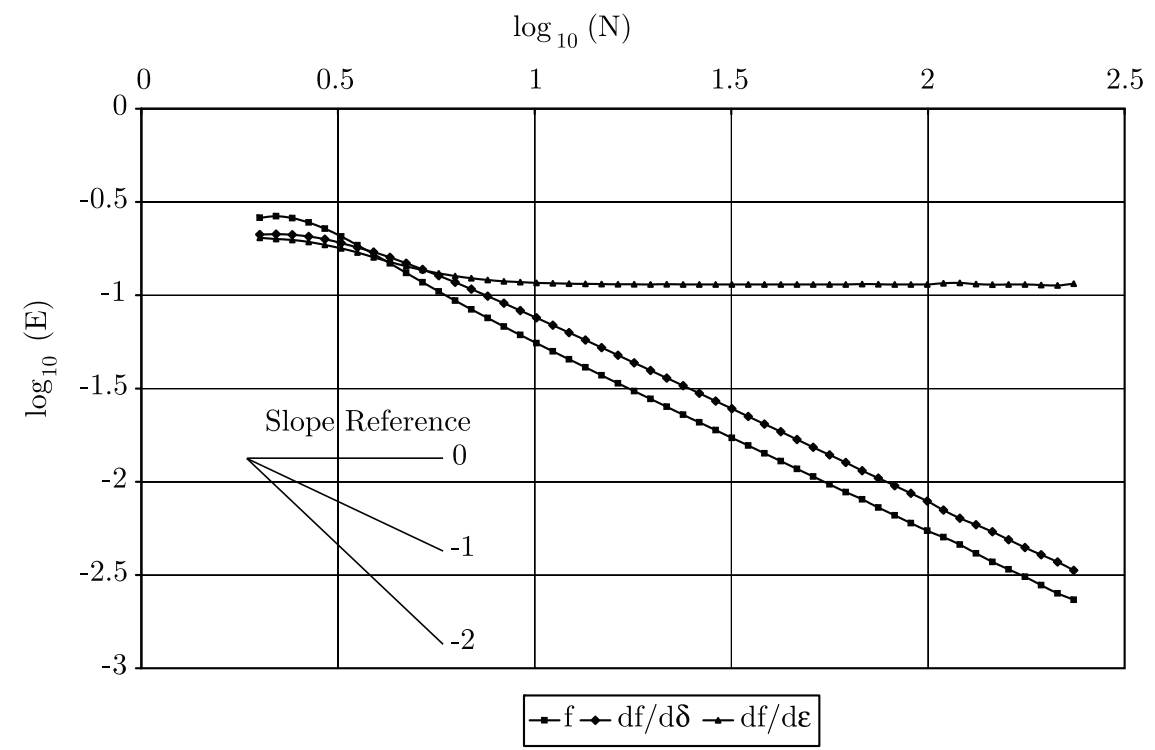

FIGURE 15. Unstructured bilinear interpolation test results on a mesh containing case $\mathrm{H}$ edges. Plots of $E_{f}, E_{d f / d \delta}$ and $E_{d f / d \varepsilon}$ are shown. Statistics are only computed over the vertical edges of the mesh whose end nodes are internal.

If the same numerical test is repeated using hybrid unstructured bilinear interpolation, then the results are as shown in Figure 16. Note that when using the hybrid scheme, the vertical edges of the mesh in Figure 14 are classified as case D rather than case $\mathrm{H}$. It can be seen that:

- The $E_{f}$ curve has a gradient of approximately -2 over a wide range of scales, indicating second order accuracy. This is consistent with the theoretical prediction for case $\mathrm{D}$ edges.

- The $E_{d f / d \varepsilon}$ curve has a gradient of approximately -1 over a wide range of scales, indicating first order accuracy. This is consistent with the theoretical prediction for case $\mathrm{D}$ edges.

- The $E_{d f / d \delta}$ curve has a gradient of approximately - 1 over a wide range of scales, indicating first order accuracy. This is consistent with the theoretical prediction for case $\mathrm{D}$ edges.

- At very small values of $N$, all three curves depart slightly from the expected straight line relationship. This is difficult to explain, but minor.

This test case is of critical importance, as it shows that the first order function interpolation accuracy demonstrated for the conventional unstructured bilinear scheme is improved to second order accuracy when the hybrid version is used. The accuracy of the $\varepsilon$ derivative is also improved from zeroth to first order accuracy. 


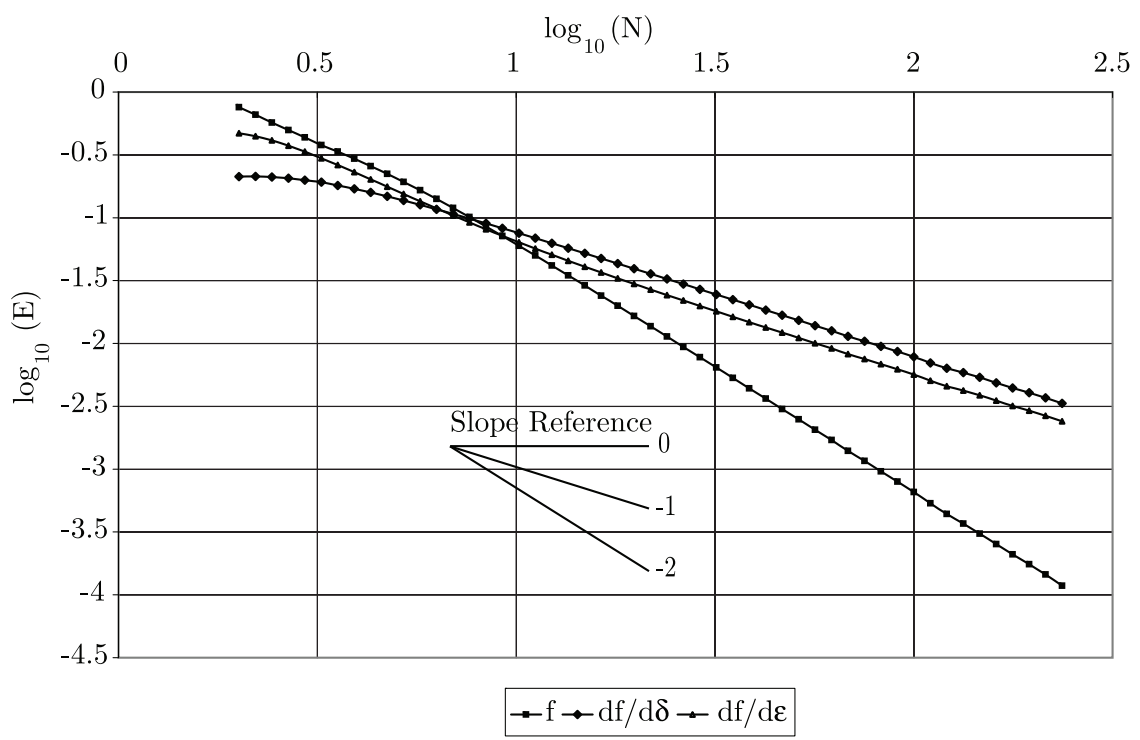

Figure 16. Hybrid unstructured bilinear interpolation test results on a mesh containing case D edges. Plots of $E_{f}, E_{d f / d \delta}$ and $E_{d f / d \varepsilon}$ are shown. Statistics are only computed over the vertical edges of the mesh whose end nodes are internal.

\section{CONCLUSION}

In this paper, it was shown that the interpolation scheme used by Kim and Choi [5] is only first order accurate for function interpolation (and zeroth order accurate for spatial derivatives), rather than being second order accurate as Kim and Choi claimed. However, Kim and Choi's results may be explained by the fact that there are numerous special cases of mesh geometry where the order of accuracy is higher than for the general case, as well as many situations where the interpolation is approximately second order accurate. Many such special cases were identified in this paper, although the list is not claimed to be comprehensive. The variety of special cases is surprising, and highlights the complexity of the interaction between the mesh geometry and the interpolation scheme, which ultimately has a very significant effect on the interpolation accuracy. Having identified the problems with Kim and Choi's interpolation scheme, this paper then presented an improved version that is consistently second order accurate for function interpolation. This new scheme is also consistently first order accurate for spatial derivatives, apart from some special cases where it is second order accurate.

\section{REFERENCES}

1. S. K. M. Chenoweth and J. Soria and A. Ooi, A singularity-avoiding moving least squares scheme for two-dimensional unstructured meshes, Journal of Computational Physics, vol. 228 (2009), pp. 5592-5619. MR2541467 (2010g:76090)

2. Y. Coudiere and J. Vila and P. Villedieu, Convergence rate of a finite volume scheme for a two dimensional convection-diffusion problem, Mathematical Modelling and Numerical Analysis, vol. 33 (1999), pp. 493-516. MR.1713235(2000f:65086) 
3. L. Davidson, A pressure correction method for unstructured meshes with arbitrary control volumes, International Journal for Numerical Methods in Fluids, vol. 22 (1996), p. 265.

4. R. L. Hardy, Multiquadric equations of topography and other irregular surfaces, Journal of Geophysical Research, vol. 76 (1971), pp. 1905-1915.

5. D. Kim and H. Choi, A second-order time-accurate finite volume method for unsteady incompressible flow on hybrid unstructured grids, Journal of Computational Physics, vol. 162 (2000), pp. 411-428. MR.1774263 (2001c:76086)

6. Y. G. Lai, An unstructured grid method for a pressure-based flow and heat transfer solver, Numerical Heat Transfer, Part B: Fundamentals, vol. 32 (1997), p. 267.

7. P. Lancaster and K. Salkauskas, Surfaces generated by moving least squares methods, Mathematics of Computation, vol. 37 (1981), pp. 141-158. MR616367 (83c:65015)

8. K. Mahesh, A family of high order finite difference schemes with good spectral resolution, Journal of Computational Physics, vol. 145 (1998), pp. 332-358. MR1644274 (99d:76068)

9. D. Shepard, A two-dimensional interpolation function for irregularly spaced points, Proceedings of the $23^{r d}$ National Conference, ACM (1968), pp. 517-523.

P.O. Box 1026, Salisbury, South Australia, S108, Australia

E-mail address: samuelchenoweth@gmail.com

Laboratory for Turbulence Research in Aerospace and Combustion, Department of Mechanical Engineering, Monash University, Victoria, 3800, Australia

E-mail address: julio.soria@eng.monash.edu.au

Department of Mechanical Engineering, University of Melbourne, Victoria, 3010, Australia

E-mail address: a.ooi@unimelb.edu.au 